\title{
Shared electrophysiology mechanisms of body ownership and motor imagery
}

\author{
Nathan Evans ${ }^{a, b}$, Olaf Blanke ${ }^{a, b, c, *}$ \\ a Center for Neuroprosthetics, School of Life Sciences, École Polytechnique Fédérale de Lausanne, Switzerland \\ ${ }^{\mathrm{b}}$ Laboratory of Cognitive Neuroscience, Brain Mind Institute, School of Life Sciences, École Polytechnique Fédérale de Lausanne, Switzerland \\ c Department of Neurology, University Hospital Geneva, Switzerland
}

\section{A R T I C L E I N F O}

Article history:

Accepted 4 September 2012

Available online 18 September 2012

Keywords:

Body ownership

Motor imagery

Mu-band

Rubber hand illusion

\begin{abstract}
A B S T R A C T
Although we feel, see, and experience our hands as our own (body or hand ownership), recent research has shown that illusory hand ownership can be induced for fake or virtual hands and may be useful for neuroprosthetics and brain-computer interfaces. Despite the vast amount of behavioral data on illusory hand ownership, neuroimaging studies are rare, in particular electrophysiological studies. Thus, while the neural systems underlying hand ownership are relatively well described, the spectral signatures of body ownership as measured by electroencephalography (EEG) remain elusive. Here we induced illusory hand ownership in an automated, computer-controlled manner using virtual reality while recording 64-channel EEG and found that illusory hand ownership is reflected by a body-specific modulation in the mu-band over fronto-parietal cortex. In a second experiment in the same subjects, we then show that mu as well as beta-band activity in highly similar fronto-parietal regions was also modulated during a motor imagery task often used in paradigms employing non-invasive brain-computer interface technology. These data provide insights into the electrophysiological brain mechanisms of illusory hand ownership and their strongly overlapping mechanisms with motor imagery in fronto-parietal cortex. They also highlight the potential of combining high-resolution EEG with virtual reality setups and automatized stimulation protocols for systematic, reproducible stimulus presentation in cognitive neuroscience, and may inform the design of non-invasive brain-computer interfaces.
\end{abstract}

(c) 2012 Elsevier Inc. All rights reserved.

\section{Introduction}

Human self-consciousness has become an increasingly prominent issue in the cognitive neurosciences in recent years (Blanke and Metzinger, 2008; Christoff et al., 2011; Gallagher, 2000). Whereas earlier research focused mainly on higher-level aspects such as memory, personality, or language and how these functions relate to the self and self-consciousness (Gillihan and Farah, 2005; Legrand and Ruby, 2009; Northoff et al., 2006), recent studies have started to investigate more basic aspects of self-consciousness, especially how we experience and perceive our body. Such mechanisms of bodily self-consciousness consist of brain mechanisms encoding the different multisensory and sensorimotor states of the body (Berlucchi and Aglioti, 1997, 2009; Botvinick, 2004; Damasio, 2000; Jeannerod, 2006, 2007; Vogeley and Fink, 2003).

One aspect that has been investigated intensively over the last decade is the experience that our body and its parts belong to us and are not those of other people, so-called body ownership. Ownership for one's hand has been proposed to constitute a crucial aspect of bodily selfconsciousness (De Vignemont, 2011; Gallagher, 2000; Makin et al., 2008; Tsakiris, 2010) and an increasing number of empirical data on

\footnotetext{
* Corresponding author at: Faculty of Life Sciences, École Polytechnique Fédérale de Lausanne, Station 19, CH-1015 Lausanne, Switzerland.

E-mail address: olaf.blanke@epfl.ch (O. Blanke).
}

the neural underpinnings of body ownership have pointed to the importance of multisensory integration of visual, tactile and proprioceptive signals (Botvinick, 2004; Botvinick and Cohen, 1998; Ehrsson et al., 2005; Tsakiris and Haggard, 2005). A widely used paradigm to study the multisensory perception of upper limbs is the rubber hand illusion (RHI; Botvinick and Cohen, 1998) where participants watch an artificial hand (visual cue) being stroked by a paintbrush in synchrony with stroking on their own corresponding and occluded hand (tactile cue). This visuo-tactile manipulation alters bodily experience, inducing the illusion that the artificial hand being touched is one's own hand (measured by questionnaire ratings) and is generally associated with a measurable mislocalization of the participant's hand towards the fake hand. The illusion does not occur when the stroking provided to the real hand and the artificial hand is not synchronous, when the fake hand does not match the posture of the real hand, or when control objects are stroked (Botvinick and Cohen, 1998; Ehrsson et al., 2004; Tsakiris and Haggard, 2005).

To investigate the brain mechanisms of illusory hand ownership, most neuroimaging studies have manipulated the synchrony of experimenter-applied visuo-tactile stroking and the congruence of posture or handedness of the fake and real hands. Using fMRI, illusory ownership as induced by synchronous visuo-tactile stroking on congruent fake hand postures was found to be reflected by BOLD activity in bilateral premotor cortices (Ehrsson et al., 2004), cerebellum (Ehrsson et al., 2005), and intraparietal cortices (Ehrsson et al., 
2004, 2005). If the fake hand is threatened by bringing a needle near to it, other studies found that activity in the supplementary motor area (Ehrsson et al., 2007) and posterior parietal regions (Lloyd et al., 2006) reflects illusory ownership. In addition, activity in bilateral anterior insular and anterior cingulate cortices (Ehrsson et al., 2007) or activity in premotor cortex and cerebellum (Ehrsson et al., 2004) was found to correlate with the strength of ownership illusion (as measured by questionnaire ratings). In a PET study, Tsakiris et al. (2007) reported that activity in the right posterior insula, sensorimotor cortices (precentral and postcentral gyri), as well as primary somatosensory cortex was associated with illusory hand ownership. Moreover, activity in the right insula and left somatosensory cortex correlated with the magnitude of proprioceptive drift (Tsakiris et al., 2007), a phenomenon classically associated with illusory hand ownership (Botvinick and Cohen, 1998; but see also Rohde et al., 2011). Finally, clinical studies in stroke patients showed a relationship between lesion location and damaged connections between premotor, frontal operculum, basal ganglia, parietal, and prefrontal cortices with the inability to experience illusory ownership for a fake hand (Zeller et al., 2011). To summarize, neuroimaging studies across a variety of RHI setups and imaging techniques (e.g. fMRI, PET, lesion mapping) have revealed a wide network of brain regions associated with illusory body ownership during the RHI. These regions include the intraparietal cortex, primary somatosensory cortex (precentral and postcentral gyri), the ventral premotor cortex, the right insular lobe, the anterior cingulate cortex, and the cerebellum (Ehrsson et al., 2004, 2005, 2007; Lloyd et al., 2006; Tsakiris et al., 2007; Zeller et al., 2011).

Concerning electrophysiological correlates of illusory hand ownership, several EEG studies using somatosensory evoked potentials (SEPs) or frequency analysis have also been carried out. For example, Kanayama et al. $(2007,2009)$ reported that gamma-band oscillations over parietal scalp regions varied according to the strength of illusory hand ownership in a RHI-like paradigm. These authors observed an increase in inter-electrode synchrony in the lower gamma-band (30-50 Hz) over parietal scalp regions during the integration of tactile and visual cues in peripersonal space. In an ERP study, Press et al. (2008) showed enhancement of the N140 and late somatosensory SEP components (evoked by hand tapping) after a period of synchronous stroking of a rubber hand, likely reflecting activation in somatosensory regions of the parietal cortex and/or premotor cortex. Related work using a different illusory hand ownership paradigm (numbness illusion) measured SEPs and implicated primary somatosensory cortex (Dieguez et al., 2009) based on the observation that the earliest cortical SEP component after median nerve stimulation (N20 component) was enhanced and correlated in strength with illusory ownership. Across these electrophysiological studies employing diverse experimental procedures, these data reveal that premotor and parietal cortex activity as well as gamma-band oscillations have most consistently been linked to illusory hand ownership.

Yet, in a number of related sensorimotor tasks, neural oscillations over central areas including premotor, motor, and somatosensory cortices have been linked rather to the mu rhythm $(8-13 \mathrm{~Hz}$ oscillations). Sensorimotor tasks (Pineda, 2005), motor action execution, inhibition, and observation (Gastaut, 1952; Howe and Sterman, 1972; Niedermeyer and Lopes da Silva, 1993) are reflected in such mu oscillations. Additionally, both intracranial electrophysiology (Gastaut and Bert, 1954; Mukamel et al., 2010; Tremblay et al., 2004) and surface EEG (Cochin et al., 1999, 1998) consistently show comparable mu rhythm suppression during both the execution and the observation of different movements. Mu oscillations have also been investigated with respect to motor imagery (review in Neuper et al., 2006) and have been linked to mu suppression in parietal cortex, premotor, and primary sensorimotor areas (Pfurtscheller and Neuper, 1997; Pfurtscheller et al., 1997a). These oscillations during hand motor imagery have also been decoded online in non-invasive brain-computer interfaces (Pfurtscheller et al., 1997b). The mu rhythm is also modulated by touch (Pfurtscheller, 1981), the observation of touch of another person
(Cheyne et al., 2003), and covaries with the BOLD signal in dorsal premotor, inferior parietal, and primary somatosensory cortices during both action execution and observation (Arnstein et al., 2011). More recently, it has also been shown that changes in body ownership for a full body as seen in a virtual reality environment are reflected in mu-activity in premotor, sensorimotor, and medial prefrontal cortices (Lenggenhager et al., 2011).

To summarize, despite this frequential and anatomical convergence of illusory hand ownership and hand motor imagery, the spatial and spectral relationship between motor imagery and illusory hand ownership has not been studied directly in the same individuals. Moreover, hand motor imagery is often used in non-invasive brain-computer interfaces (e.g. Pfurtscheller and Neuper, 2001) and it has recently been speculated that illusory ownership over virtual and prosthetic limbs may benefit neuroprosthetics and neuro-rehabilitation (Ehrsson et al., 2008; Marasco et al., 2011). Here, we designed a virtual reality environment with automatized, machine-controlled visuo-tactile stimulation to induce changes in illusory hand ownership while recording 64-channel EEG. Using this setup, we first analyzed cortical oscillations and their neural generators reflecting changes in illusory body ownership. Next, we investigated - in the same subjects - brain oscillations and their neural generators during a hand motor imagery paradigm (e.g. Pfurtscheller et al., 1997b) and directly compared ownership-related brain activations with oscillations present during motor imagery.

\section{Materials and methods}

\section{Participants}

12 healthy, right-handed participants were recruited (ages $22.7 \pm 4.1$ mean $\pm S D$; 3 females). All participants had normal or corrected-tonormal vision and gave informed consent prior to participation. The study was undertaken in accordance with the ethical standards as defined in the Declaration of Helsinki and was approved by the local ethics research committee at the University of Lausanne.

\section{Tactile stimulation}

Tactile stimulation was provided with a total of eight button-style vibration motors (Precision Microdrives, London, UK) affixed in a line to the palms of the participants' hands (Fig. 1A). On each hand, a custom-made set of four vibration motors (12 mm diameter; $1.7 \mathrm{~g}$; maximum rotation frequency $150 \mathrm{~Hz}$ ) was placed with an inter-vibrator distance of $2 \mathrm{~cm}$. The motors were programmed to vibrate in sequence to simulate a continuous, stroke-like movement lasting $450 \mathrm{~ms}$ ( $75 \mathrm{~ms}$ per motor; $50 \mathrm{~ms}$ inter-motor vibration pause). This type of sequence was chosen to automatize the stroking patterns that are generally used to manually stroke participants' hands to induce the RHI (i.e. Botvinick and Cohen, 1998; Ehrsson et al., 2004). The direction of the stroking sequence was either inward, toward a central fixation cross (6 subjects), or outward, away from the fixation cross (6 subjects). An inter-stroke interval of $400 \mathrm{~ms}$ was inserted between strokes to aid in perceiving the sequence of vibrations as a single motion.

\section{Stimuli and virtual reality}

Visual stimuli were rendered in stereo (XVR; VRMedia, Pisa, Italy) on a Fakespace Wide5 head-mounted display (HMD; Fakespace Labs, Mountain View, CA, USA). The HMD displayed a virtual scene with either two virtual arms or two virtual non-body control objects visually projected as extending from the body and resting on a tabletop (Figs. 1B, C). Four virtual spheres on each palm of the two virtual arms (or two virtual control objects) visually represented the four vibration motors on the real hands. Visual "vibrations" were represented by changing the virtual motor's color from white to red and animating it to visually jitter between 

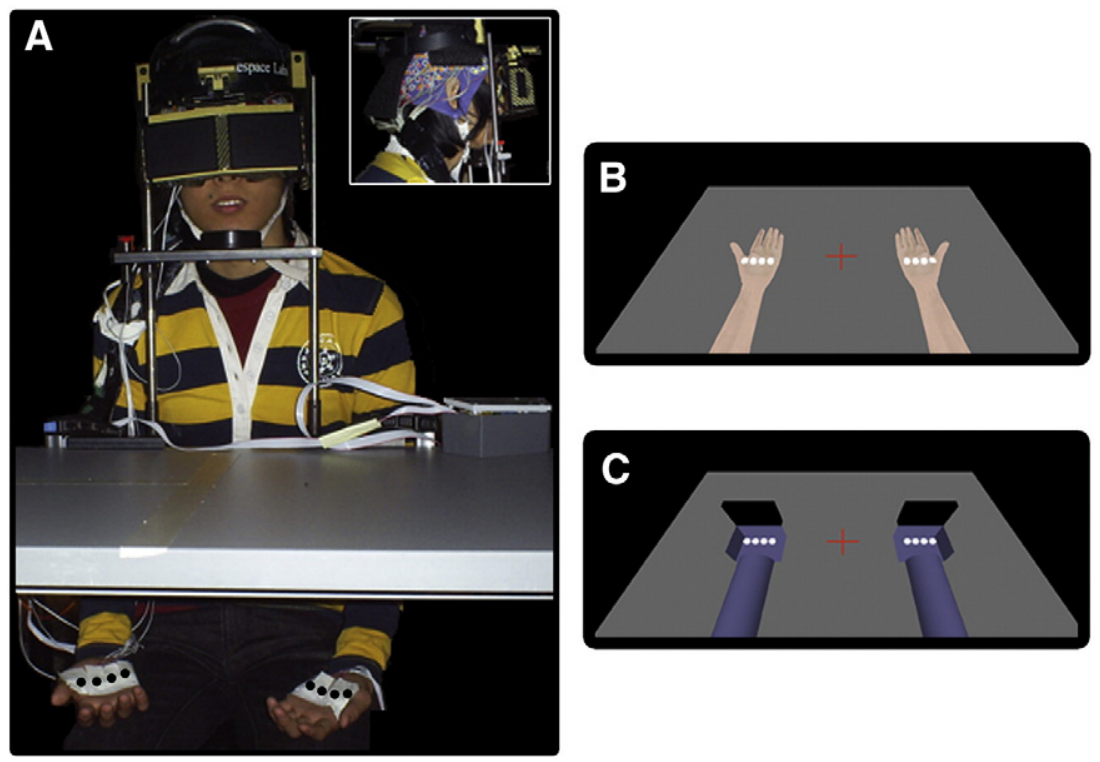

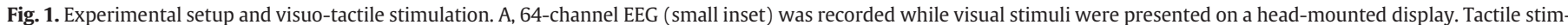

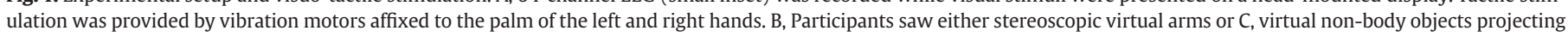
from their body onto a virtual table $(\sim 20 \mathrm{~cm}$ above where they felt their hands to be on their laps).

$\pm 1 \mathrm{~cm}$ from its original position for the entire duration of the physical vibration.

\section{Procedure}

Participants were seated at a table with their arms resting (palms up) on their legs (Fig. 1A). Head movements were restrained with a chin rest and the experiment took place in a darkened room. The HMD fully blocked the subject's vision of the table, the physical arms and vibrators, and the rest of the room. In order to approximately calibrate the perspective of the virtual scene to that of the physical scene, the HMD was individually fitted to each participant such that the virtual and physical tables aligned. The two virtual arms (or two virtual control objects) were projected above the participant's physical arms (elevated by approx. $20 \mathrm{~cm}$ ).

Synchronous visuo-tactile stimulation was defined such that visual and tactile vibrations occurred with no temporal delay and with spatial congruency between visual and tactile stimuli. To achieve asynchronous visuo-tactile stimulation for a given stroke (i.e. pattern of four vibrations), we inserted a random delay of 50 to $150 \mathrm{~ms}$ of the onset of the visual stimulation with respect to the onset of the tactile stimulation and randomly varied the direction of visual stroking (inward or outward; see Costantini and Haggard, 2007) while maintaining the same physical vibration sequence.

\section{Pilot study}

In a pilot study, using the same automated experimental setup as used in the main experiment, we induced changes in subjective (self-reports) and behavioral measures (proprioceptive drift) that are compatible with previous RHI experiments using unilateral visuo-tactile stimulation (see Supplementary material). In particular, our pilot results indicate that illusory hand ownership and perceived hand position were shifted toward the virtual hand for synchronous as compared to asynchronous visuo-tactile stroking (i.e. significant change in proprioceptive drift; Botvinick and Cohen, 1998; Tsakiris and Haggard, 2005). In contrast to the bilateral induction of the RHI (illusory ownership experiment) and bilateral presentation of visual hands (both illusory ownership and motor imagery experiments), the pilot study involved unilateral induction of the RHI. In the main experiments, we only measured subjective responses (illusory body ownership) as we were mostly interested in illusory hand ownership and its relation to motor imagery, and because we wished to avoid augmenting the already long experimental protocol.

\section{Hand ownership}

Following the behavioral pilot study confirming that our automatized and virtual reality setup induces comparable changes in hand ownership to those described in previous RHI studies using manually applied stroking, we carried out an EEG study to investigate the neural mechanisms of illusory hand ownership. We manipulated the strength of illusory hand ownership using a $2 \times 2$ factorial design with the factors Stroking and Object. The Stroking factor consisted of two levels of visuo-tactile stimulation (synchronous and asynchronous; as defined above). The Object factor was composed of two levels: virtual arms or virtual non-body control objects. An additional baseline condition was recorded, resulting in a total of five conditions. Each experimental condition was repeated three times and their presentation order was randomized and balanced within and across subjects.

Participants were asked to fixate on a cross that was placed centrally between the hands/objects located at a visual angle of $37^{\circ}$ to the left and right. For $10 \mathrm{~s}$, participants received visuo-tactile stimulation according to one of the experimental conditions. For the baseline condition, no tactile stimulation was provided to the hands and EEG was recorded at rest as participants fixated between the hands/objects as in the experimental conditions. To quantify hand ownership (Botvinick and Cohen, 1998; Slater et al., 2008) participants were asked to verbally respond to four questions (on a 1-7 Likert scale) immediately following visuo-tactile stimulation. The four questions were projected on the virtual table: 1) It seemed as if I were feeling the vibrations in the location where I saw the virtual hands being vibrated, 2) It seemed as though the vibrations I felt were caused by the vibrations I saw on the virtual hands, 3) I felt as if the virtual hands were my own hands, and 4) I felt as if my (real) hands were moving or drifting towards the virtual hands' position.

\section{Motor imagery}

We wished to additionally investigate how the neural mechanisms behind motor imagery of hand movements overlap with the neural systems activated during the experience of hand ownership. 
Participants were asked to fixate on a green fixation cross that was shown for $500 \mathrm{~ms}$ and was located centrally between the hands or the control objects. The fixation cross was briefly replaced by a centrally presented left or right arrow cue for $500 \mathrm{~ms}$ and finally replaced by another central fixation cross in red that was shown for $4.5 \mathrm{~s}$. While fixating on the red fixation cross, participants were asked to imagine clasping and unclasping the cued hand (right or left hand; Pfurtscheller et al., 1997b). It is important to note that while performing motor imagery, participants received the same visual scene as during the RHI procedure (bilateral visual hand stimuli; e.g. Neuper et al., 2009). Each experimental block consisted of 30 randomized trials ( 15 left hand imagery, 15 right hand imagery trials) and was repeated three times, resulting in 90 trials.

\section{EEG: Preprocessing}

64-channel EEG was sampled at $2048 \mathrm{~Hz}$ (Biosemi Inc, Amsterdam, Netherlands), downsampled to $512 \mathrm{~Hz}$, and subjected to visual inspection in the time and frequency domains (e.g. Tadi et al., 2009). Electrodes with $>50 \mu \mathrm{V}$ DC-offset were rejected. The mean percentage of electrodes included was 92\% ( $59 \pm 1$ electrodes; mean $\pm S D$ ). Timeframes with eye blinks and transient conductance shifts were marked as artifacted in a semi-automated manner (see Lenggenhager et al., 2011).

To analyze the RHI data, thirty total seconds of recorded EEG per condition were broken into $2 \mathrm{~s}$ epochs $(14 \pm 1 \mathrm{~s}$; mean $\pm S D$ per subject, per condition). For motor imagery, 2 min of total EEG data was collected for the period immediately following the imagery cue and was further broken into $2.25 \mathrm{~s}$ epochs ( $60 \pm 19$ epochs; mean $\pm S D$ per subject, per condition). Note that the motor imagery EEG data were part of a larger study (Evans \& Blanke; unpublished results), leading to the difference in number of data epochs analyzed for RHI and motor imagery periods. To maximize usage of non-artifacted data, epochs were fit with $25 \%$ overlap between timeframes marked as artifacted (see above). Prior to computing power spectral densities (PSD), scalp potentials were re-referenced with respect to the average reference, the linear trend was removed, and a Hann window was applied to each epoch (Blackman and Tukey, 1959). PSDs were then computed at a $0.5 \mathrm{~Hz}$ resolution for each epoch with a Fast Fourier Transform (Matlab, Mathworks, Natick, Massachusetts, USA) in the following three frequency bands alpha/mu $(8-13 \mathrm{~Hz})$, beta (14-25 Hz) and gamma (25-55 Hz). Finally, PSD values for each subject $s$, experimental condition $c$, frequency band $f$, and electrode $e$, $P_{e}(s, c, f)$, were averaged across epochs and a log power ratio (LPR) was computed by taking the logarithm of the result of dividing each PSD by the subject's mean baseline PSD, $M_{e}(s, f)$, (i.e. Oberman et al., 2005). The LPRs, $L_{e}(s, c, f)$, thus took the form:

$L_{e}(s, c, f)=\ln \left(P_{e}(s, c, f)\right)-\ln \left(M_{e}(s, f)\right)$

\section{EEG: Statistical analysis}

\section{Hand ownership during visuo-tactile stimulation}

Single electrode analysis. We analyzed EEG signals recorded at electrodes C3 and C4, located over left and right hand sensorimotor cortex (Oberman et al., 2005; Pfurtscheller and Neuper, 1994). These electrodes were selected on the basis of the hypothesized location of the overlap between brain activity associated with illusory hand ownership and hand motor imagery (e.g. Kanayama et al., 2009; Munzert et al., 2009) and because somatosensory evoked potentials at electrodes C3 and C4 have been shown to be modulated by illusory ownership (Peled et al., 2003; Press et al., 2008). We note, however, that EEG changes at scalp electrodes C3/C4 may result from neural generators at close and distant locations in the brain (Michel and Murray, 2012). LPRs were contrasted across the four experimental conditions using a $2 \times 2$ repeated-measures ANOVA and were further analyzed with post-hoc t-tests corrected for multiple comparisons (Bonferroni correction).

Cluster analysis. Next, we searched for scalp electrodes where LPRs reflected changes in illusory ownership. For this, we first determined for each electrode, each experimental condition, and each frequency band whether the LPRs significantly differed from the baseline condition (i.e. LPR of 0) using two-tailed, paired t-tests (see Oberman et al., 2005). This resulted in one scalp distribution of p-values for each experimental condition (Bonferroni corrected for multiple comparisons; Maris and Oostenveld, 2007). In the case when electrodes had been rejected during preprocessing for one or more subjects (see EEG: Preprocessing section), the statistical tests were performed with fewer measurements (i.e. a smaller sample size). Neighboring significant electrodes were then formed into spatial clusters by taking the union of the sets of all significant electrodes across the four experimental conditions and requiring that each member electrode have at least two neighbors (for a similar method, see Lenggenhager et al., 2011; Mitsis et al., 2008). This clustering technique resulted in the formation of clusters of electrodes that significantly differ from the baseline condition in at least one, but not necessarily all of the experimental conditions. Potential neighbors were defined as electrodes falling within a radius of $3 \mathrm{~cm}$ and, under this definition, the mean number of neighbors per electrode was 4 . Electrode labels for all significant electrodes and the resulting clusters in the mu band $(8-13 \mathrm{~Hz})$ are presented in Table 1 . Finally, for each experimental condition, a per-subject LPR was computed within each cluster by taking the mean LPR of the cluster's member electrodes. Statistical differences between experimental conditions were gaged using a $2 \times 2$ repeated-measures ANOVA on the distribution of LPRs across subjects. We additionally analyzed whether subjective questionnaire ratings for the ownership question (item 3) correlated (Pearson correlation) with the per-subject, mean LPRs in the electrode clusters for mu-, beta-, and gamma-bands.

\section{Motor imagery}

Motor imagery has been shown to exhibit lateralized effects over sensorimotor cortex, particularly in the mu- and beta-bands (Pfurtscheller and Neuper, 1997), and also in the gamma band (Miller et al., 2010; Pfurtscheller and Neuper, 2001). To verify whether our paradigm and experimental setup using automated stroking and virtual reality was also associated with these EEG changes, we analyzed LPRs in mu, beta, and gamma bands over left and right sensorimotor scalp regions (at the

\section{Table 1}

Significant electrodes used in whole-scalp cluster analyses. Electrode labels (10-20 system) for scalp electrodes surviving statistical correction in the mu-band $(8-13 \mathrm{~Hz})$ for the illusory hand ownership and motor imagery of hands studies. Contiguous, significant, individual electrodes were then grouped, resulting in two sensorimotor clusters (see Materials and methods section).

\begin{tabular}{|c|c|c|}
\hline & Individual electrodes & Clustered \\
\hline \multicolumn{3}{|l|}{ Illusory ownership } \\
\hline Body sync & C6, CP6, T8 & Left cluster: \\
\hline Body async & C3, C4, T8, СР6 & $\mathrm{C} 1, \mathrm{C} 3, \mathrm{CP} 1, \mathrm{CP} 3$ \\
\hline \multirow[t]{2}{*}{ Object sync } & $\mathrm{C} 1, \mathrm{C} 3, \mathrm{CP} 1, \mathrm{CP} 3$, & \\
\hline & $\begin{array}{c}\text { FT8, FC6, C4, CP4, } \\
\text { CP6 }\end{array}$ & $\begin{array}{l}\text { Right cluster: } \\
\text { C4, C6, CP4, CP6 }\end{array}$ \\
\hline Object async & $\mathrm{C} 4, \mathrm{C} 6$ & \\
\hline \multicolumn{3}{|l|}{ Motor imagery } \\
\hline \multirow[t]{2}{*}{ Left imagery } & $\begin{array}{l}\text { C1, C3, C5, СР1, } \\
\text { P9, PO7, C4, C6, } \\
\text { CP6, CP2, P2, P4, }\end{array}$ & $\begin{array}{l}\text { Left cluster: } \\
\text { C1, C3, CP1, C5 }\end{array}$ \\
\hline & $\begin{array}{c}\mathrm{P} 6, \mathrm{PO} 4 \\
\mathrm{C} 1, \mathrm{C} 3, \mathrm{C} 5, \mathrm{CP} 1\end{array}$ & $\begin{array}{l}\text { Right cluster: } \\
\text { C4, C6, CP6, }\end{array}$ \\
\hline Right imagery & $\begin{array}{c}\text { P9, PO7, C4, C6, } \\
\text { CP6, CP2, P2, P4, } \\
\text { P6, PO4 }\end{array}$ & $\begin{array}{c}\mathrm{CP} 2, \mathrm{P} 2, \mathrm{P} 4, \mathrm{P} 6, \\
\mathrm{PO} 4\end{array}$ \\
\hline
\end{tabular}


level of single electrodes, C3 and C4, and at the cluster level) during left and right motor imagery. We used these classical neurophysiological responses to motor imagery as a biomarker that participants performed the motor imagery task. No further questionnaire or behavioral measures related motor imagery process was performed, because the experiment was already long. As a baseline condition, we calculated the average of left and right motor imagery on a per-electrode (scalp) or per-voxel (inverse solution) basis. Statistical differences were assessed using a $2 \times 2$ repeated-measures ANOVA with the factors Imagery (left or right hand) and Electrode side (left or right scalp hemisphere). Finally, post-hoc, two-tailed t-tests were used to further analyze any main effects or interactions (Bonferroni corrected).

\section{Source localization}

Cortical neural generators for scalp potentials were computed with a pseudo-inverse of the electrical lead field (sLORETA; Pascual-Marqui, 2002) using a head model extracted from a "standard" brain template (MNI152; Fuchs et al., 2002). Raw EEG data were preprocessed as in the scalp-level analysis. Electrodes previously rejected from scalp level analysis were replaced with a linear interpolation. Cross-spectra were computed in mu-, beta- and gamma-bands, were averaged persubject and per-condition, and an inverse transformation matrix was applied to the cross spectra. Finally, an F-ratio was computed at the voxel level to statistically contrast each experimental condition to the baseline condition, as performed in an earlier work on body ownership (e.g. Lenggenhager et al., 2011) and related work (Oberman et al., 2005; Pineda, 2005). This contrast was also used for our analysis at the level of the scalp electrodes. Statistical maps were log transformed, participantwise normalized, and corrected for Type I errors (SnPM; Nichols and Holmes, 2002). All reported coordinates are in MNI (Montreal Neurological Institute) space.

We additionally wished to verify that differences between the body synchronous and body asynchronous conditions observed at the single trace and cluster levels remained consistent at the voxel level. For this, we first determined two spherical ROIs based on the contrasts obtained separately from the inverse solution for the body synchronous and the body asynchronous conditions versus the baseline condition. The center of each ROI was defined as the left and right hemispheric centroids of the union of all supra-threshold voxels (F-ratio $<-0.175$ ) and the volume of the ROI defined by a $10 \mathrm{~mm}$ radius. The mean log power across voxels in these two ROIs was then determined for both body conditions.

\section{Overlap analysis}

To assess spectral and spatial commonalities between hand ownership and hand motor imagery at the scalp level, a meta-set of electrodes was composed for both study 1 and for study 2 . To build these two electrode meta-sets, the union of the clustered electrodes from each study was taken (c.f. Table 1, rightmost column). These clustered electrodes originated from the set of electrodes differing from the baseline condition in at least one, but not necessarily all experimental conditions from each respective study.

We analyzed similarities in the spectral profiles by taking the per-subject peak power frequency from the mean power spectra in the two meta-sets. Differences in peak power frequency were separately assessed for the left and right hemispheres using paired, two-tailed t-tests. Next, we computed spatial overlap at the scalp level by counting overlapping electrodes from the two meta-sets separately in the left and right hemispheres.

A similar method was used to compute spatial overlap at the voxel level. Supra-threshold voxels were taken from the respective inverse solutions using the same mask (F-ratio<-0.175) for each experimental condition. In order to then compare the significant voxels associated with motor imagery with those associated with illusory hand ownership in each of the four experimental conditions, we collapsed left and right motor imagery data sets into a single data set of significant voxels. To do so, we applied the supra-threshold mask to the inverse solutions for both left and right imagery and then took the union of the significant voxels from these two sets. Left hemispheric (MNI X coordinate $<0$ ) and right hemispheric (MNI X coordinate $>0$ ) overlaps were quantified separately by counting the number of overlapping and non-overlapping voxels. To assess the overall overlap in each experimental condition from the illusory hand ownership study, the mean of the left and right hemispheric overlap was taken, resulting in one percentage overlap value per condition.

\section{Results}

Self-reported illusory hand ownership

A Friedman non-parametric ANOVA revealed a significant effect of the experimental condition on subjective ratings for each item of the questionnaire $\left(\mathrm{Q} 1: \chi^{2}{ }_{(12,3)}=18.82 ; \mathrm{Q} 2: \chi^{2}{ }_{(12,3)}=16.78 ; \mathrm{Q} 3\right.$ : $\chi^{2}{ }_{(12,3)}=20.01 ; \mathrm{Q} 4: \chi^{2}{ }_{(12,3)}=20.87$; all $\left.\mathrm{p}<0.001\right)$. Fig. 2 shows that illusory hand ownership (Q3) was largest in the synchronous, body stroking condition (henceforth referred to as illusion condition). Post-hoc, non-parametric Wilcoxon matched pair tests (all comparisons were Bonferroni corrected) revealed, as predicted, that only the illusion condition significantly differed from all other conditions (all $\mathrm{p}<0.01$ ). Importantly, only the illusion condition resulted in positive illusory hand ownership and no significant difference was found between the non-body control objects ( $p>0.05$ ).

For items 1 and 2, post-hoc Wilcoxon matched pair tests revealed a significant difference between synchronous and asynchronous stroking for both body and non-body objects (both $\mathrm{p}<0.01$ ). No significant difference in subjective ratings between body and non-body objects was observed for synchronous (both $\mathrm{p}>0.10$ ) or for asynchronous stroking (both $\mathrm{p}>0.10$ ). For item 4 , the post-hoc tests showed a significant difference between synchronous and asynchronous stroking for bodily objects $(\mathrm{p}<0.02)$, but no such difference for non-body objects $(\mathrm{p}>0.10)$. The full questionnaire results are plotted in Supplemental Fig. S1.

\section{Electrophysiology of illusory hand ownership}

\section{Single electrode analysis (electrodes $\mathrm{C} 3 / \mathrm{C} 4$ )}

For mu-band activity at electrode $\mathrm{C} 4$, a repeated-measures ANOVA revealed a significant two-way interaction between Synchrony and Object $\left(\mathrm{F}_{1,11}=6.43, \mathrm{p}<0.05\right)$ but no main effects of Synchrony or Object (both $\mathrm{p}>0.20$ ). The mu-band power in the illusion condition was found to be less strongly suppressed than in the remaining conditions and thus differed significantly from the asynchronous body condition $(\mathrm{p}<0.01)$ as

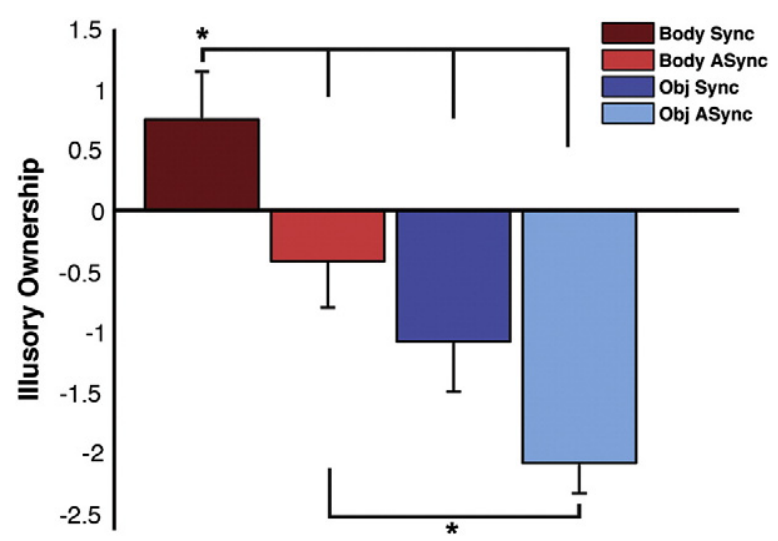

Fig. 2. Illusory hand ownership. Mean questionnaire scores probing the subjective experience of illusory ownership during visuo-tactile stimulation (Question 3; error bars SEM). Two types of stimulation (synchronous, asynchronous) were performed on two different visual objects (hands or control objects). * indicates a significant difference between experimental conditions ( $\mathrm{p}<0.05$; Wilcoxon matched pairs test; corrected). 
well as the synchronous object condition ( $p<0.05$; Fig. 3B). No such difference was found between synchronous and asynchronous stroking for the non-body control objects ( $p>0.05$ ). At electrode $\mathrm{C} 3$, mu-band power did not show any significant interaction or main effects (all $\mathrm{p}>0.10$ ).

In beta- and gamma-bands at electrode C4, no significant main effects or interactions were found (all $\mathrm{p}>0.05$ ). Beta and gamma-band analysis at electrode $\mathrm{C} 3$ revealed no interaction or main effect of $\mathrm{Ob}$ ject (all $\mathrm{p}>0.05$ ), but a main effect of Synchrony in the beta-band $\left(\mathrm{F}_{1,11}=8.58 ; \mathrm{p}<0.01\right)$ and the gamma-band $\left(\mathrm{F}_{1,11}=4.73, \mathrm{p}<0.05\right)$. A full summary of the mean LPRs is presented in Table 2.

\section{Cluster analysis}

For each experimental condition, at least one electrode (mean: 5) was found to significantly differ from the baseline condition in the mu-band (Table 1; left column). Using our clustering technique, we found the electrode clusters (assembled across the experimental conditions; see Materials and methods section) to be localized to scalp regions over right and left sensorimotor cortex (Fig. 3A; left cluster: 4 electrodes, right cluster: 4 electrodes; Table 1, right column). Statistical analysis for the right mu-band cluster revealed a two-way interaction between Synchrony and Object $\left(\mathrm{F}_{1,11}=6.59, \mathrm{p}<0.05\right)$ but no main effects (both $\mathrm{p}>0.2$ ). Mu-band power in the illusion condition was found to be less strongly suppressed than the remaining conditions and to be significantly different than the asynchronous body condition ( $\mathrm{p}<0.05$; Fig. $3 \mathrm{C}$ ) as well as the synchronous object condition $(p<0.05)$. No such difference was found between synchronous and asynchronous stroking for the non-body control objects ( $p>0.05$ ). For the left cluster, a trend towards significance was found for the interaction term $(p=0.09)$ without any main effects (all p>0.35). Beta and gamma-band analysis in both left and right mu-defined clusters did not reveal any significant interactions or main effects (all $\mathrm{p}>0.05$ ). The mean mu-band LPRs in the left and
A
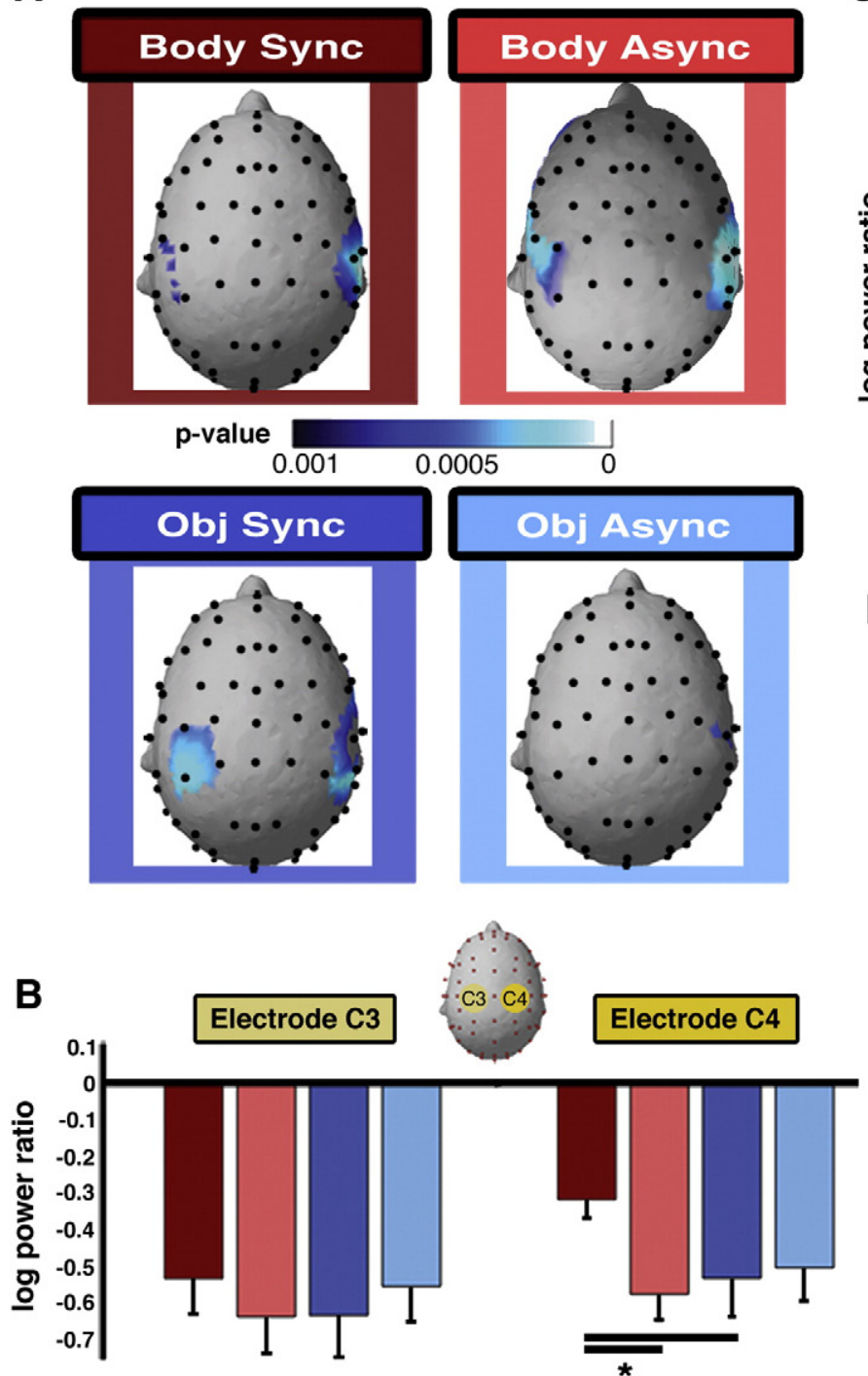

C

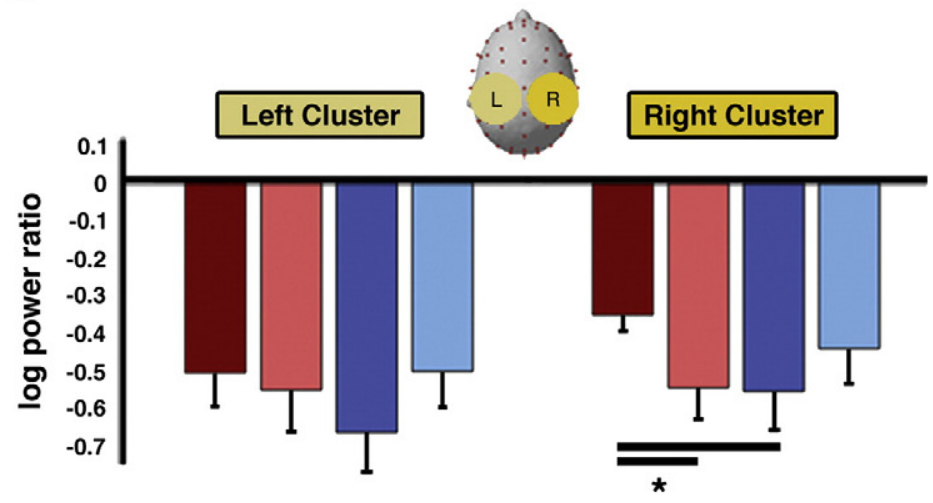

D

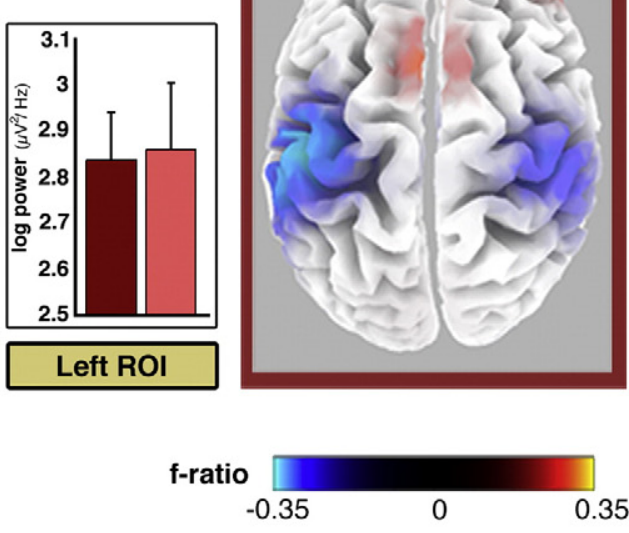

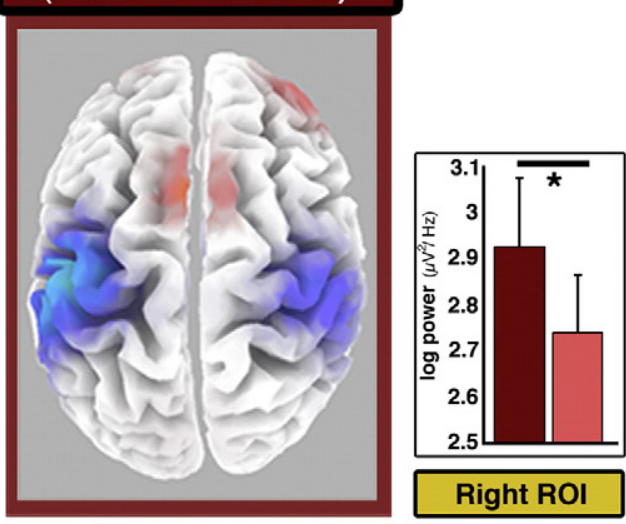

Body Sync (Illusion Condition)

$-0.35$ 0.35

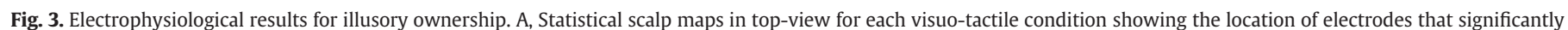

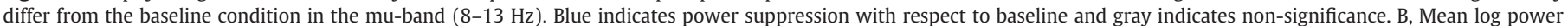

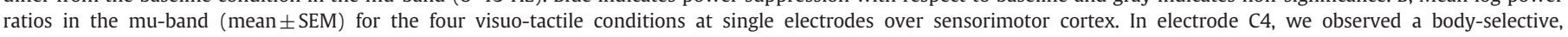

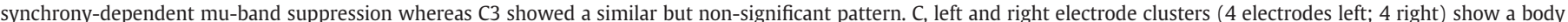

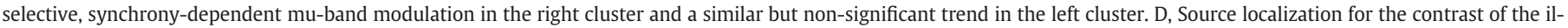

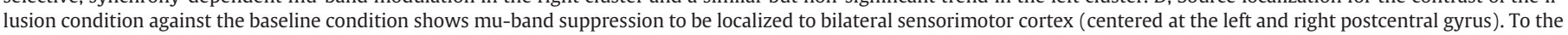
left and right, log power is plotted in voxel-level ROIs (as defined in Materials and methods section) for body synchronous and body asynchronous conditions. 
right clusters for the illusion condition were not found to correlate with the questionnaire responses for question 3 (all p >0.05). In the beta and gamma bands, no electrodes were found to significantly differ from the baseline condition. Thus, no electrode clusters were defined for beta- or gamma-bands.

\section{Source localization}

The mu-band suppression found at the single electrode and cluster levels in the illusion condition was localized to the sensorimotor cortex and extended from premotor to posterior parietal cortex. Its maximal focus was found at the right and left postcentral gyrus (right: $\mathrm{F}=-0.35$; Brodmann areas $3 / 4$; peak MNI coordinates: $\mathrm{X}=40$, $\mathrm{Y}=-25, \mathrm{Z}=55$; left: $\mathrm{F}=-0.30$; Brodmann areas $3 / 4$; peak MNI coordinates: $X=-45, Y=-20, Z=55$ ).

To confirm the log power difference observed at the single trace and cluster levels at the voxel level, we defined two ROIs (see Materials and methods section). The left hemispheric ROI had its centroid at $X=-41$, $\mathrm{Y}=-26, \mathrm{Z}=49$ and the right $\mathrm{ROI}$ at $\mathrm{X}=40, \mathrm{Y}=-28, \mathrm{Z}=45$. In agreement with single trace and cluster analyses, log power was found to differ between the body synchronous and body asynchronous conditions in the right hemispheric ROI $(\mathrm{p}<0.05)$ but not in the left hemispheric ROI ( $p>0.05$ ). In Fig. 3D, log power for the synchronous body condition and asynchronous body conditions is plotted for both ROIs and is plotted alongside the inverse solution for the illusion condition.

In summary, illusory hand ownership was reflected in the suppression of mu-band power at the postcentral gyrus extending into premotor cortex and posterior parietal cortex. These localized oscillatory modulations were absent for control conditions under otherwise identical visuo-tactile conditions. Beta and gamma-band activity did not reflect illusory ownership, visuo-tactile synchrony, or the sight of a virtual arm at the cluster level. Rather, these frequency bands were modulated by visuo-tactile synchrony at scalp electrode $\mathrm{C} 3$, and were not found to be body-specific.

\section{Motor imagery of hands}

Using single trace (electrodes C3, C4), cluster, and source localization analyses, we next studied whether mu, beta and gamma-band power yields contralateral suppression (event-related desynchronization) and ipsilateral enhancement (event-related synchronization) during lateralized motor imagery tasks (Pfurtscheller and Neuper, 1997; Pfurtscheller et al., 1997b) and how this compares to the observed changes in illusory hand ownership. For this, we applied a $2 \times 2$ repeated-measures ANOVA with the factors Imagery (left or right imagery) and Electrode side (left or

\section{Table 2}

Log power ratios for each experimental condition in single trace and cluster analyses. Mean and standard error of log power ratios (illusory ownership) and log power (motor imagery). Values are provided for single trace analyses (electrodes C3 and C4) as well as cluster analyses (left and right clusters; see Materials and methods section, Figs. 3 and 4).

\begin{tabular}{lrrrr}
\hline & Electrode C3 & Electrode C4 & Left cluster & Right cluster \\
\hline $\begin{array}{l}\text { Illusory ownership } \\
\text { (mu) }\end{array}$ & & & & \\
Body sync & $-0.53 \pm 0.10$ & $-0.31 \pm 0.05$ & $-0.51 \pm 0.09$ & $-0.35 \pm 0.05$ \\
Body async & $-0.64 \pm 0.10$ & $-0.57 \pm 0.07$ & $-0.55 \pm 0.11$ & $-0.54 \pm 0.08$ \\
Object sync & $-0.63 \pm 0.11$ & $-0.53 \pm 0.10$ & $-0.66 \pm 0.10$ & $-0.55 \pm 0.10$ \\
$\quad$ Object async & $-0.55 \pm 0.10$ & $-0.50 \pm 0.09$ & $-0.50 \pm 0.10$ & $-0.44 \pm 0.09$ \\
Motor imagery (mu) & & & & \\
$\quad$ Left imagery & $0.14 \pm 0.04$ & $-0.20 \pm 0.05$ & $0.10 \pm 0.04$ & $-0.18 \pm 0.05$ \\
$\quad$ Right imagery & $-0.22 \pm 0.05$ & $0.13 \pm 0.04$ & $-0.17 \pm 0.06$ & $0.13 \pm 0.04$ \\
Motor imagery & & & & \\
$\quad$ (beta) & & & & \\
Left imagery & $0.06 \pm 0.02$ & $-0.13 \pm 0.04$ & $0.04 \pm 0.01$ & $-0.12 \pm 0.03$ \\
Right imagery & $-0.11 \pm 0.03$ & $0.09 \pm 0.03$ & $-0.06 \pm 0.02$ & $0.08 \pm 0.03$ \\
\hline
\end{tabular}

right hemisphere). Table 2 summarizes the mean log powers in the left and right motor imagery conditions.

Single electrode analysis (electrodes $\mathrm{C} 3 / \mathrm{C} 4$ )

A repeated-measures ANOVA on the mu-band LPRs showed a significant two-way interaction between Imagery and Electrode side $\left(\mathrm{F}_{1,11}=25.89, \mathrm{p}<0.01\right)$ without significant main effects (both $\mathrm{p}>0.5$ ). Further testing revealed that this was caused by a significant difference in mu-band power between contralateral and ipsilateral motor imagery at $\mathrm{C} 3(\mathrm{p}<0.01)$ and at $\mathrm{C} 4(\mathrm{p}<0.01$; Fig. $4 \mathrm{C})$ and, in particular, a stronger suppression in mu-band power at $\mathrm{C} 3$ for right imagery (compared to right imagery at $\mathrm{C} 4 ; \mathrm{p}<0.01$ ) and at $\mathrm{C} 4$ for left imagery (compared to left imagery at $\mathrm{C} 3 ; \mathrm{p}<0.01$ ).

In the beta-band, the same pattern was observed as in the mu-band, namely a significant two-way interaction $\left(F_{1,11}=15.77, p<0.01\right)$ and a significant difference between contralateral and ipsilateral motor imagery at electrodes $\mathrm{C} 3(\mathrm{p}<0.01)$ and $\mathrm{C} 4(\mathrm{p}<0.01)$. As for mu-band power, beta-band power was also significantly suppressed for right motor imagery in electrode C3 (as compared to $\mathrm{C} 4 ; \mathrm{p}<0.01$ ) and suppressed for left motor imagery in electrode C4 (as compared to C3; $\mathrm{p}<0.01$ ).

In the gamma-band, statistical analysis also revealed a two-way interaction $\left(\mathrm{F}_{1,11}=10.43, \mathrm{p}<0.01\right)$. Only a significant difference between ipsilateral and contralateral band power in electrode $\mathrm{C} 3(\mathrm{p}<0.01)$ was observed, whereas no such difference was present in electrode C4 ( $p>0.1$ ). Following the pattern of mu- and beta-bands, gamma-band power was significantly suppressed for right motor imagery in electrode C3 (as compared to C4; $\mathrm{p}<0.01$ ) and suppressed for left motor imagery in electrode $\mathrm{C} 4$ (as compared to $\mathrm{C} 3$; $\mathrm{p}<0.05$ ).

\section{Cluster analysis}

For left and right motor imagery, 14 electrodes were found to significantly differ from the baseline condition in the mu-band. These electrodes were clustered at scalp regions over left and right sensorimotor cortex (compare Fig. 3A with Fig. 4A; left cluster: 4 electrodes and right cluster: 8 electrodes; see Table 1 ). For the beta-band, we found the same clusters (14 electrodes, yielding two clusters: 4 left and 8 right). No electrodes were found to significantly differ from the baseline condition for left or right motor imagery in the gamma-band.

A repeated-measures ANOVA on the cluster LPRs showed a two-way interaction in the mu-band (Imagery $\times$ Electrode side; $F_{1,11}=14.31$, $\mathrm{p}<0.01$ ). Post-hoc analysis showed that in both clusters, contralateral mu-band power significantly differed from ipsilateral mu-band power (left cluster: $\mathrm{p}<0.01$; right cluster: $\mathrm{p}<0.01$; Fig. 4D). As expected, this difference was due to a stronger suppression of mu-band power in the left cluster for right imagery (compared to right imagery in the right cluster; $\mathrm{p}<0.01$ ) and a stronger suppression in the right cluster for left imagery (compared to left imagery in the left cluster; $\mathrm{p}<0.01$ ). The same pattern was found in the beta-band (Imagery $\times$ Electrode side interaction; $F_{1,11}=13.71, p<0.01$ ), where contralateral band power significantly differed from ipsilateral band power (left cluster: $\mathrm{p}<0.01$; right cluster: $\mathrm{p}<0.01$ ). As observed in the mu-band, beta-band power was suppressed contralaterally: in the left cluster for right imagery (as compared to right imagery in the right cluster; $\mathrm{p}<0.01$ ) and in the right cluster for left imagery (as compared to left imagery in the left cluster; $\mathrm{p}<0.01$ ).

\section{Source localization}

During left motor imagery, mu-band power suppression was localized to right premotor and primary motor cortices $(F=-0.31$; Brodmann areas 4/6; peak MNI coordinates: $X=35, Y=-15, Z=70$ ) and mu-band power enhancement to left premotor and primary motor cortices $(F=0.20$; Brodmann areas $4 / 6$; peak MNI coordinates: $\mathrm{X}=-45, \mathrm{Y}=-15, \mathrm{Z}=50$; see Fig. $4 \mathrm{~B}$, left). The opposite was found for right motor imagery: suppression in left premotor and primary motor cortices $(F=-0.27$; Brodmann areas 4/6; peak MNI coordinates: $\mathrm{X}=-45, \mathrm{Y}=-15, \mathrm{Z}=50$ ) and mu-band power enhancement in right 
A

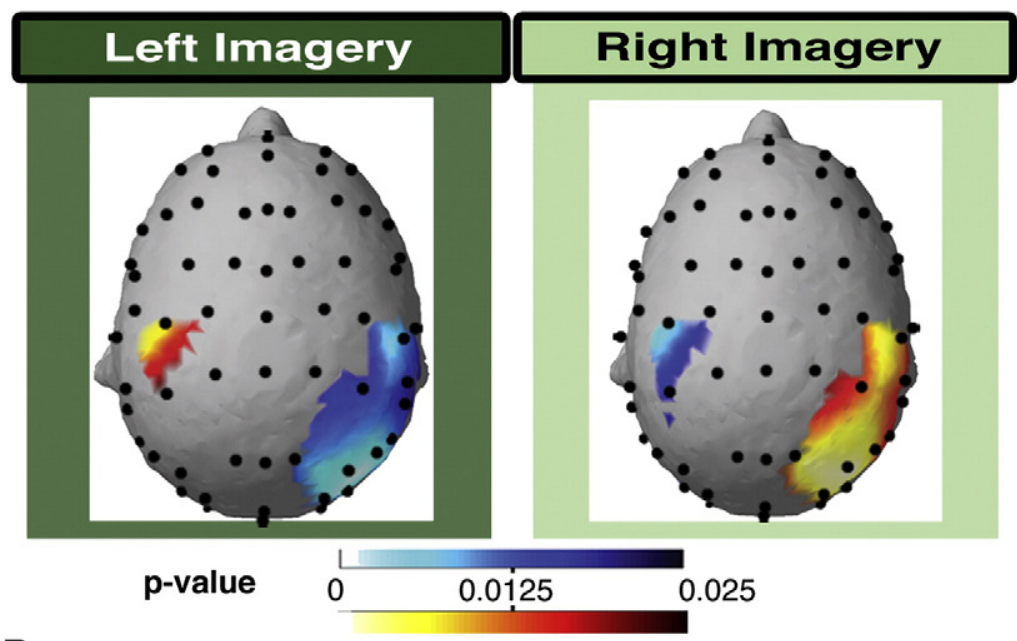

B

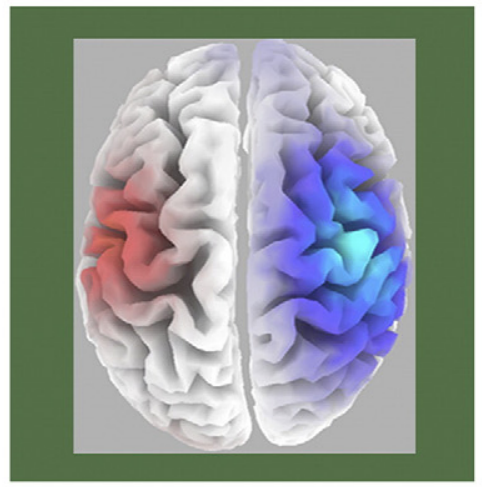

f-ratio

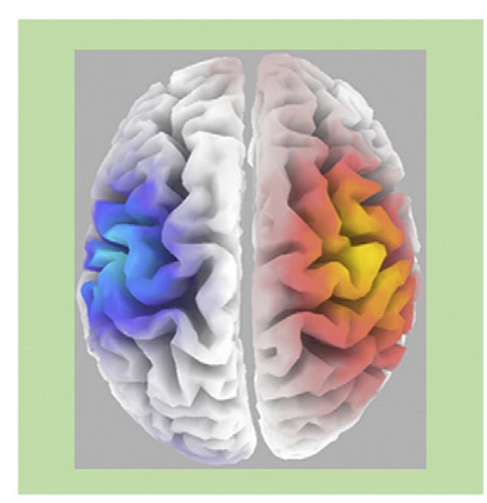

$-0.31$

0

0.31

C
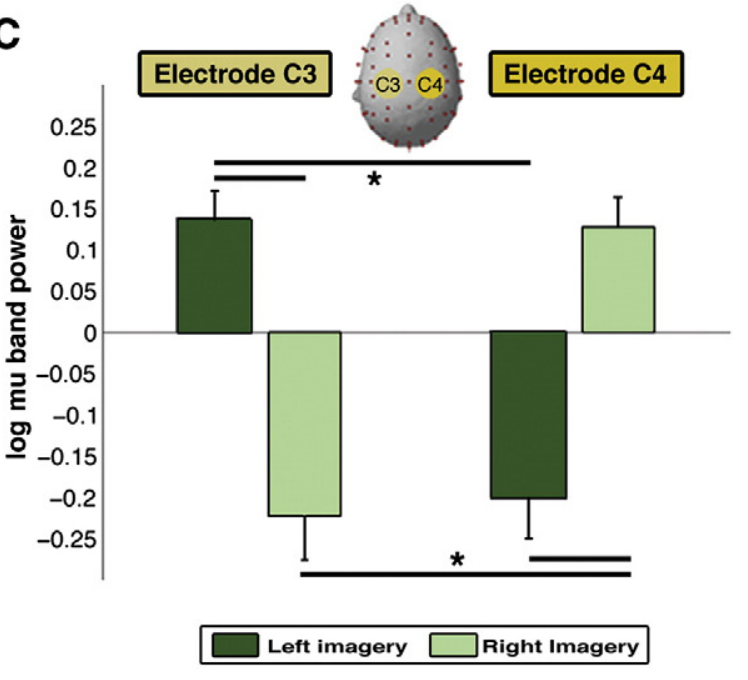

D

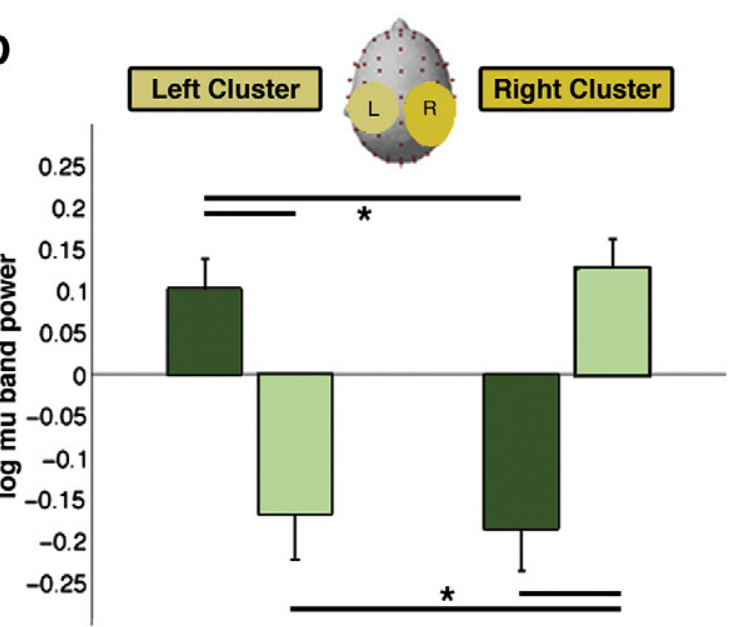

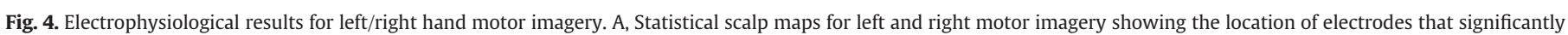

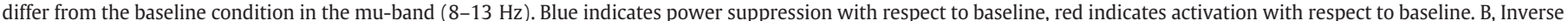

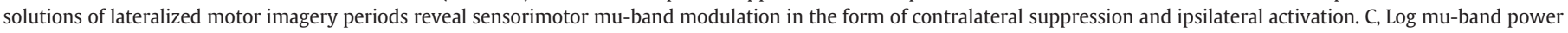

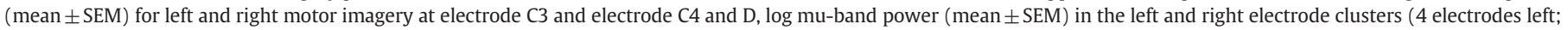
8 right) showed significant differences across imagery side (left/right) and hemisphere (left/right).

premotor and primary motor cortices $(F=0.25$; Brodmann areas $4 / 6$; peak MNI coordinates: $X=40, Y=-10, Z=65$; see Fig. $4 B$, right).

Contralateral beta-band suppression was found for both left and right motor imagery and was centered in precentral gyrus, extending from premotor to parietal areas (left imagery: $F=-0.08$; Brodmann area 6; peak MNI coordinates: $X=30, Y=-15, Z=50$; right imagery: $\mathrm{F}=-0.07$; Brodmann area 6; peak MNI coordinates: $\mathrm{X}=-35, \mathrm{Y}=-5$, $Z=45$ ); ipsilateral enhancement was also found for both left and right imagery and was focused at the middle frontal gyrus extending from premotor to parietal regions (left imagery: $\mathrm{F}=-0.06$; Brodmann area 6 ; peak MNI coordinates: $X=-35, Y=-5, Z=60$; right imagery: $\mathrm{F}=-0.08$; Brodmann area 6; peak MNI coordinates: $\mathrm{X}=35, \mathrm{Y}=0$, $\mathrm{Z}=65)$.

\section{Spatial and spectral overlap between ownership and motor imagery}

These results suggest that lateralized motor imagery as well as illusory hand ownership is reflected by mu-band oscillations in sensorimotor cortex, including premotor and posterior parietal cortices. To further investigate this finding, we looked at the spectral profile across subjects for ownership and motor imagery, contrasting peak power responses in the frequency domain and further investigated the anatomical overlap between the two sets of activations at the scalp and inverse solution levels.

This analysis revealed that the spectral profile of mu-suppression was the same for motor imagery and illusory ownership as tested here; by contrasting the per-subject peak spectral power in the left hemisphere, we found no significant difference between peak power frequency during illusory ownership $(10.0 \pm 0.42 \mathrm{~Hz}$; mean \pm SEM) and motor imagery ( $9.6 \pm 0.36 \mathrm{~Hz} ; \mathrm{p}>0.05)$. Moreover, no peak power response difference was observed in the right hemisphere between these two processes (ownership: $9.7 \pm 0.3 \mathrm{~Hz}$; motor imagery: $10.1 \pm 0.3 \mathrm{~Hz}$; p $>0.05$ ).

Concerning spatial overlap at the scalp level, 6 of 8 (mean: 75\%) of the significant electrodes found in the cluster analysis for illusory ownership overlapped with significant electrodes found during motor imagery (full summary in Table 3). In Fig. 5, the inverse solutions for illusory hand ownership, right hand imagery, and left hand imagery are overlaid, showing strong spatial overlap in voxel space (mean: $86.9 \%$; percentage overlap for illusory ownership and motor imagery). Computing overlap for each experimental condition showed that the illusion condition led 
to the strongest overlap with motor imagery (mean across hemispheres: $89 \%$ ) as compared to the body asynchronous and object synchronous conditions (mean across hemispheres: 78\% and 72\%, respectively). The weakest overlap was found for the object-asynchronous condition (49\%).

\section{Discussion}

Here we induced and manipulated systematic changes in illusory hand ownership for two virtual hands using a fully automated setup combining tactile stimulation, virtual reality and electrical neuroimaging. Mu-band activity, but not beta or gamma-band activity, reflected illusory hand ownership and these changes were localized to fronto-parietal cortex. These data provide novel insights into the brain mechanisms of body ownership and related aspects of bodily self-consciousness, and highlight the potential of combining high-resolution EEG with virtual reality setups and automatized stimulation protocols for systematic, reproducible stimulus presentation in neuroscience (i.e. Bohil et al., 2011; Lenggenhager et al., 2011; Slater et al., 2008). Mu-band activity in highly similar regions was also modulated during hand motor imagery. Although premotor and posterior parietal cortices have been involved in illusory hand ownership and motor imagery respectively, the present study is the first to show common mechanisms of these two processes in these regions and in the mu band, within the same subjects.

Hand ownership as studied in the RHI has been shown to be sensitive to various parameters including visuo-tactile stroke duration (Rohde et al., 2011; Tsakiris and Haggard, 2005), distance between proprioceptive and visual hand position (Lloyd, 2007), and visual hand posture (Costantini and Haggard, 2007). In the large majority of these studies, the experimenter applied the visuo-tactile stroking manually (but see also Schütz-Bosbach et al., 2009) and to one hand. Here we describe an automatized and programmed RHI setup that makes the different stimulation-related parameters explicit and reproducible across the different visuo-vibratory conditions. These well-defined spatio-temporal parameters should facilitate the comparison and development of future behavioral and neuroimaging studies related to illusory hand ownership, for instance to those that have already been applied in the field of neuro-prosthetics (Marasco et al., 2011). Moreover, an automated RHI setup overcomes previous experimental constraints as it allowed us, for instance, to simultaneously apply visuo-tactile stimulation to both hands. Using this novel set-up, we have shown that comparable effects can be achieved when using automatized visuo-tactile stimulation in an immersive virtual reality scenario with virtual hands projecting from one's shoulders in stereo vision.

Concerning illusory referral of touch (questions 1 and 2), our setup was effective and induced strong illusions of touch that were dependent on the synchrony of stroking. Yet, this was not found to be body-specific

\section{Table 3}

Anatomical overlap between illusory ownership and motor imagery. Illusory ownership data (illusion condition) is spatially compared to motor imagery (see Materials and methods section). Percentage overlap is quantified separately in the left and right hemisphere for significant, mu-band modulated scalp electrodes and voxels. Top half: The number of overlapping scalp electrodes (from left and right sensorimotor electrode clusters as defined in Table 1) between illusory hand ownership and motor imagery of hands. Bottom half: The number of overlapping voxels (from inverse solution contrasts; see Results section for voxel coordinates).

\begin{tabular}{cll}
\hline & Imagery with ownership & Ownership with imagery \\
\hline $\begin{array}{c}\text { Scalp clusters (electrodes) } \\
\text { Left hemisphere }\end{array}$ & $\begin{array}{l}3 \text { of } 4 \\
(75 \%)\end{array}$ & $\begin{array}{l}3 \text { of } 4 \\
(75 \%)\end{array}$ \\
Right hemisphere & 3 of 4 & 3 of 8 \\
& $(75 \%)$ & $(38 \%)$ \\
Source generators (voxels) & & 198 of 367 \\
Left hemisphere & 198 of 201 & $(54 \%)$ \\
Right hemisphere & $(98.5 \%)$ & 258 of 336 \\
& 258 of 324 & $(76.8 \%)$ \\
\hline
\end{tabular}

(as in Armel and Ramachandran, 2003; Lenggenhager et al., 2007) and could in the present study be due to the fact that the non-body objects were similar in size and form to the hands (for discussion see Tsakiris, et al., 2009). We also note that many of the aforementioned RHI studies (e.g. Botvinick and Cohen, 1998; Ehrsson et al., 2004; Tsakiris and Haggard, 2005) did not collect self-reports for non-body control objects. Concerning illusory hand ownership (item 3), the present self-reports provide evidence that such changes can be induced in a body-specific and synchrony-dependent fashion with our automated setup (see also Slater et al., 2008). We observed similar effects also for illusory drift in hand position (question 4). Accordingly, the present questionnaire data extend and complement earlier work that has studied illusory ownership over virtual hands presented on a distanced projection screen (Slater et al., 2008), on a monitor (Hägni et al., 2008), or via videoprojector (Ijsselsteijn et al., 2006) as well as more traditional RHI studies (Botvinick and Cohen, 1998; Tsakiris and Haggard, 2005).

\section{Fronto-parietal regions reflect ownership}

Previous neuroimaging work not only revealed the prominent involvement of premotor and intraparietal cortices in illusory hand ownership, but also implicated other brain regions such as primary somatosensory cortex, insular cortex, anterior cingulate cortex, and the cerebellum (Ehrsson et al., 2004, 2005, 2007; Lloyd et al., 2006; Tsakiris et al., 2007; Zeller et al., 2011). The present inverse solution data link illusory hand ownership to bilateral premotor and posterior parietal cortices (Ehrsson et al., 2004, 2005, 2007) as well as the postcentral gyrus (Ehrsson et al., 2005; Lloyd et al., 2006; Schaefer et al., 2006a, 2006b; Tsakiris et al., 2007; see also Schaefer et al., 2007).

Concerning the lateralization of brain activation, our data suggest a predominant involvement of the right fronto-parietal cortex. This is difficult to compare with prior neuroimaging studies on the RHI as these have adopted a wide range of tactile stimulation protocols. Most authors manually applied visuo-tactile stroking either unilaterally to the right (Costantini and Haggard, 2007; Ehrsson et al., 2004, 2005, 2007; Lloyd, 2007; Lloyd et al., 2006; Tsakiris et al., 2007), or unilaterally to the left dorsum and fingers of the hand (Botvinick and Cohen, 1998; Kanayama et al., 2007, 2009; Schaefer et al., 2006a, 2006b; Tsakiris and Haggard, 2005). We extended the diversity of the employed RHI protocols and stimulated the palm bilaterally in an automatized fashion, confirming and extending previous findings on illusory hand ownership. Furthermore, these different stimulation protocols resulted in distributed, but partially overlapping patterns of brain activity associated with illusory hand ownership. In previous reports, unilateral visuo-tactile stroking led to brain activity contralateral (Lloyd et al., 2006; Schaefer et al., 2006a, 2006b; Tsakiris et al., 2007), bilateral (Ehrsson et al., 2004, 2005, 2007; Lloyd et al., 2006; Press et al., 2008), or ipsilateral with respect to the stimulated hand (Lloyd et al., 2006; Tsakiris et al., 2007). Using EEG source analysis and bilateral visuo-tactile stimulation, we observed activity in bilateral fronto-parietal regions. Despite the bilateral pattern of activation at the level of the inverse solution, however, statistical analyses at the single electrode and cluster levels only revealed significant effects over right fronto-parietal regions (whereas the same analysis was not significant at C3 or the left cluster). The present findings are therefore in favor of a selective or predominant right hemispheric involvement in illusory hand ownership and are consistent with a behavioral study reporting stronger illusory ownership in the left hand as opposed to the right hand (Ocklenburg et al., 2010). This right lateralization of hand ownership is further supported by the prevalence of right-hemispheric lesions leading to the neuropsychological condition of somatoparaphrenia, during which patients report abnormal ownership for their left contralesional limb (for a review see Vallar and Ronchi, 2009; Karnath and Baier, 2010). Future research should investigate differences between unilateral and bilateral visuo-tactile stimulations as well as differences between 

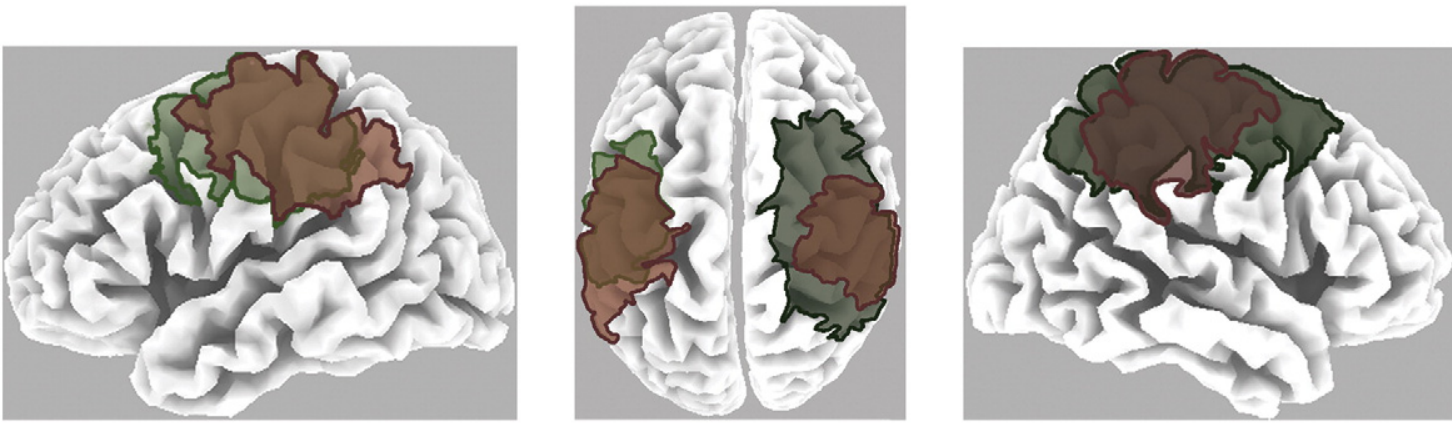

Right Imagery $\square$ Illusory Ownership $\square$ Left Imagery

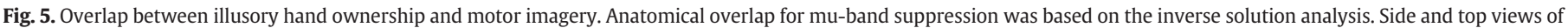

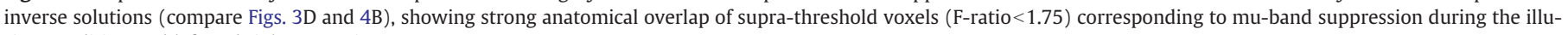
sion condition and left and right motor imagery.

manually versus automated visuo-tactile stimulation and how these influence the recruitment of right versus left fronto-parietal cortex.

Previous clinical studies using overlap analysis in stroke patients also suggested that fronto-parietal damage in insular cortex could be responsible for variable dysfunctions in limb ownership including somatoparaphrenia (e.g. Karnath and Baier, 2010). Such an implication of the insula has also been found for illusory hand ownership by experimentally manipulating visuo-tactile information in healthy subjects (Tsakiris et al., 2007), although the insular activity in this study correlated only with implicit measures of the RHI (proprioceptive drift) and not with subjective changes in illusory hand ownership as tested in the present study. Although we cannot exclude that insular cortex was also activated in the present study and may have influenced the observed fronto-parietal EEG patterns, this is rather unlikely given our inverse solution results and that activity in the insula can be detected with EEG methods (Mulert et al., 2003).

\section{Electrophysiology of ownership}

The present frequency analysis revealed that illusory hand ownership was reflected in mu-band suppression in fronto-parietal regions, but not beta or gamma-band oscillations in this or other regions. By simultaneously measuring EEG and PET/fMRI, sensorimotor alpha and mu-band suppression has been associated with increased cerebral activity (Goldman et al., 2002; Oakes et al., 2004). Furthermore, mu-band suppression has been linked to sensorimotor processing (Pineda, 2005), such as action execution and observation (Gastaut, 1952), tactile stimulation (Pfurtscheller, 1981), and observed tactile stimulation of others (Cheyne et al., 2003). Here, we found mu-band activity to distinguish between the illusion condition and the three control conditions. Namely, the illusion condition led to less mu-band suppression than the control conditions. We thus found greater activation of fronto-parietal cortex during both non-body and the asynchronous body conditions, concordant with previous EEG and PET data on illusory hand ownership (Lenggenhager et al., 2011; Tsakiris et al., 2007). This brain activation pattern also excludes that mere visuo-tactile matching or the sight of a virtual hand accounts for the observed mu-band changes. Increased activation, as reflected by greater suppression with respect to the baseline condition, has been proposed to be due to greater multisensory visuo-tactile conflict (Fink et al., 1999; Lenggenhager et al., 2011). In further agreement with the observed mu-band modulation in the present study, illusory hand ownership has been associated with decreased cortical excitability and cortical inhibition of the motor cortex (Schütz-Bosbach et al., 2006, 2009). Accordingly, we argue that fronto-parietal cortex is less activated and/or inhibited during synchronous visuo-tactile stimulation (illusion condition) as compared to the baseline condition, where no visuo-tactile stimulation was applied.
In a series of EEG experiments, illusion strength during the RHI was linked to an increase in inter-electrode, phase-locking synchrony in the gamma-band over parietal cortex (Kanayama et al., 2007, 2009). Though the present data mainly point to a close association of illusory hand ownership with changes in mu oscillations, our data are also in partial agreement with the data by Kanayama and colleagues. We found a main effect of visuo-tactile synchrony over central scalp regions (electrode C3, left cluster) that was reflected by beta-band suppression and an increase in gamma-band activity. Kanayama et al. (2007) reported gamma changes to be maximal over the central-parietal region (i.e. electrode Pz). However, since these authors were mainly interested in crossmodal synchrony effects during the RHI, they restricted their analysis to high-beta and low-gamma bands (20-100 Hz); frequency bands known to be associated with bimodal integration (for a review, see Calvert and Thesen, 2004). As such, no direct comparison can be made with our results that show illusory hand ownership to be selectively associated with mu-band power suppression. We note, however, that in contrast to these authors, our data do not suggest that beta or gamma-band activity is related to illusory hand ownership, because activity changes in these bands did not reflect illusory hand ownership depending on our experimental factors of stroking synchrony and the sight of virtual hands or objects. Importantly, visuo-tactile stimulation in these previous studies by Kanayama et al. (2007, 2009) was always performed on body-like objects, i.e. no object control conditions were carried out to test for body specificity of synchrony-related changes in hand ownership. We also note that the collected subjective reports in the two studies by Kanayama and colleagues suggest that most participants did not experience illusory ownership within their experimental setting, whereas in the present experimental setup, illusory ownership was significantly modulated. We thus suggest that these earlier EEG results represent a low-level effect of receiving bimodal (visuo-tactile) stimulation in a synchronous or asynchronous manner, rather than illusory hand ownership. Such comparisons, however, ought to be made cautiously, as the experimental setup and paradigm differ substantially from the current study.

Shared spectral and anatomical mechanisms between motor imagery and ownership

Our motor imagery results follow classical electrophysiological responses over sensorimotor cortex (Pfurtscheller and Neuper, 1997; Neuper et al., 2006). These are characterized by contralateral mu- and beta-band desynchronization (suppression) and ipsilateral mu- and beta-band synchronization (enhancement) and were found here at both the level of single electrode and cluster analyses. The application of a linear, distributed inverse solution localized these changes to contralateral and ipsilateral fronto-parietal cortex including premotor and 
posterior parietal cortices as well as the postcentral gyrus. These regions have been involved in a number of studies in motor imagery (see reviews from Grèzes and Decety, 2001; Munzert et al., 2009). Several studies using neuroimaging (Kosslyn et al., 2001), transcranial magnetic stimulation (Ganis et al., 2000), or clinical investigations (Sirigu et al., 1996) showed that motor imagery shares neural mechanisms with movement planning (Decety et al., 1989) and movement execution (Gerardin et al., 2000; Parsons et al., 1995), in particular in premotor cortex (e.g. Ionta et al., 2010) and parietal cortex (e.g. Overney and Blanke, 2009). Activity in both the inferior parietal cortex (Wolbers et al., 2003) and the premotor cortex (de Lange et al., 2006; Malouin et al., 2003) increases as a function of imagery demand. Of relevance for the present study, premotor cortex and posterior parietal cortex are activated in the RHI (Ehrsson et al., 2004, 2005) and activation in premotor cortex has been found to correlate with illusion strength. Moreover, RHI and motor imagery have been shown to depend on multisensory mechanisms, including vision and proprioceptive signals (Botvinick and Cohen, 1998; Ionta and Blanke, 2009; Ionta et al., 2012; Lloyd, 2007) and changes in illusory hand ownership alter the speed of motor imagery (Ionta et al., 2012). To our knowledge, our results show for the first time at the neural level a strong anatomical overlap between illusory hand ownership and hand motor imagery in the same subjects showing that common structures are recruited for both processes. That is, the activation changes due to motor imagery activated to a large extent the same regions that were modulated by illusory hand ownership with the former being somewhat more widespread than the latter. This overlap was maximal during the illusion condition and minimal during asynchronous visuo-tactile stimulation during the non-body object condition.

Common brain mechanisms between hand ownership and hand motor imagery were further confirmed using frequency analysis. Thus, concerning the involved frequency bands, both motor imagery and illusory hand ownership were associated with mu-band modulation. However, motor imagery was characterized by contralateral mu- and beta-band desynchronization in fronto-parietal cortex (suppression; i.e. Pfurtscheller and Neuper, 1997; Pfurtscheller et al., 1997b), whereas our illusory hand ownership manipulation was characterized by mu-band desynchronization, especially in the right fronto-parietal cortex, that was body-specific and depended on visuo-tactile stimulation. These frequency data suggest that hand motor imagery and illusory hand ownership rely on similar electrophysiological mechanisms in fronto-parietal cortex, while partly differing in their spectral signatures.

There are several limitations to the present study. We note that the present experiment was designed as a precursor to future work investigating the impact of illusory ownership on $\mathrm{BCI}$ performance involving bilateral presentation of the visual hands during unilateral motor imagery. This was one of the reasons why we decided to induce ownership bilaterally in the present study, measuring the spatial and spectral overlap of illusory ownership and motor imagery by comparing bilateral RHI data against a combination of two unilateral motor imagery data sets. This constraint may have been a limitation concerning the present overlap analysis, as recruited brain structures and overlap may be different from those described here if unilateral RHI data are compared with unilateral motor imagery (or if bilateral RHI data are compared with bilateral hand motor imagery).

Also, in the present study participants saw static virtual hands while performing motor imagery and this may have interfered with motor imagery mechanisms due to incongruency between the imagined movement and the static virtual hands. Recent neuroimaging evidence has also suggested that providing visual postural consequences congruent with the motor imagery strategy may enhance corticospinal activity in M1 (Mercier et al., 2008). Given this, future studies may additionally manipulate the visual feedback during motor imagery to investigate the effects of non-stationary visual feedback on brain mechanisms of motor imagery as well as the shared mechanisms of the RHI and motor imagery. Furthermore, collection of additional behavioral measures of motor imagery performance could allow for comparisons between the strength of illusory ownership and imagery ability. Further work is needed to investigate and compare the brain mechanisms of unilateral and bilateral hand ownership and motor imagery.

Collectively, these anatomical and spectral data reveal that motor imagery in fronto-parietal cortex shares neural mechanisms not only with several different motor-related aspects such as movement execution and movement planning, but also with multisensory mechanisms related to illusory hand ownership. It has been speculated that illusory ownership over virtual and prosthetic limbs may benefit neuroprosthetics and neuro-rehabilitation (Ehrsson et al., 2008; Marasco et al., 2011). The present data show that the computer-controlled induction of hand ownership alters - at least partly - the same fronto-parietal oscillations that can be used in non-invasive brain computer interfaces. These approaches have utilized motor imagery for motor control via online exploitation of spectral features from the mu- and beta-bands ( $8-26 \mathrm{~Hz}$ ) over sensorimotor cortex (Lebedev and Nicolelis, 2006; Millán et al., 2010; Pfurtscheller and Neuper, 2001; Pfurtscheller et al., 1997b; Wolpaw and McFarland, 2004). Based on the present findings, we argue that automatized illusory hand ownership may be used to guide or improve control of external devices including robotic arms using non-invasive brain computer interface technology as well as to control prosthetic arms that are interfaced with the peripheral nervous system (Marasco et al., 2011; Navarro et al., 2005).

\section{Acknowledgments}

The authors would like to thank Silvio Ionta for feedback on earlier versions of the manuscript. Support for this project was provided by the VERE project: FP7-ICT-2009-5 - Project 257695 and the Bertarelli Foundation.

\section{Appendix A. Supplementary data}

Supplementary data to this article can be found online at http:// dx.doi.org/10.1016/j.neuroimage.2012.09.027.

\section{References}

Armel, K.C., Ramachandran, V.S., 2003. Projecting sensations to external objects: evidence from skin conductance response. Proc. R. Soc. Lond. 270 (1523), 1499-1506.

Arnstein, D., Cui, F., Keysers, C., Maurits, N.M., Gazzola, V., 2011. $\mu$-suppression during action observation and execution correlates with BOLD in dorsal premotor, inferior parietal, and SI cortices. J. Neurosci. 31 (40), 14243-14249.

Berlucchi, G., Aglioti, S., 1997. The body in the brain: neural bases of corporeal awareness. Trends Neurosci. 20 (12), 560-564.

Berlucchi, G., Aglioti, S., 2009. The body in the brain revisited. Exp. Brain Res. 1-11.

Blackman, R., Tukey, J., 1959. The Measurement of Power Spectra from the Point of View of Communications Engineering. Dover, New York.

Blanke, O., Metzinger, T., 2008. Full-body illusions and minimal phenomenal selfhood. Trends Cogn. Sci. 7.

Bohil, C., Alicea, B., Biocca, F., 2011. Virtual reality in neuroscience research and therapy. Nat. Rev. Neurosci. 1-11.

Botvinick, M., 2004. Probing the neural basis of body ownership. Science 305 (5685), 782-783.

Botvinick, M., Cohen, J., 1998. Rubber hands 'feel' touch that eyes see. Nature 391 (6669), 756.

Calvert, G., Thesen, T., 2004. Multisensory integration: methodological approaches and emerging principles in the human brain. J. Physiol. Paris 98 (1-3), 191-205.

Cheyne, D., Gaetz, W., Garnero, L., Lachaux, J., Ducorps, A., Schwartz, D., 2003. Neuromagnetic imaging of cortical oscillations accompanying tactile stimulation. Cogn. Brain Res. 17 (3), 599-611.

Christoff, K., Cosmelli, D., Legrand, D., Thompson, E., 2011. Specifying the self for cognitive neuroscience. Trends Cogn. Sci. 15 (3), 104-112.

Cochin, S., Barthelemy, C., Roux, S., Martineau, J., 1999. Observation and execution of movement: similarities demonstrated by quantified electroencephalography. Eur. J. Neurosci. 11 (5), 1839-1842.

Cochina, S., Barthelemya, C., Lejeuneb, B., Rouxa, S., Martineau, J., 1998. Perception of motion and qEEG activity in human adults. Electroencephalogr. Clin. Neurophysiol. 107, 287-295.

Costantini, M., Haggard, P., 2007. The rubber hand illusion: sensitivity and reference frame for body ownership. Conscious. Cogn. 16 (2), 229-240.

Damasio, A., 2000. The Feeling of What Happens: Body and Emotion in the Making of Consciousness. Harcourt Brace, New York. 
de Lange, F., Helmich, R., Toni, I., 2006. Posture influences motor imagery: an fMRI study. Neuroimage 33 (2), 609-617.

De Vignemont, F., 2011. Embodiment, ownership and disownership. Conscious. Cogn. 20 (1), 82-93.

Decety, J., Jeannerod, M., Prablanc, C., 1989. The timing of mentally represented actions. Behav. Brain Res. 34 (1-2), 35-42.

Dieguez, S., Mercier, M., Newby, N., Blanke, O., 2009. Feeling numbness for someone else's finger. Curr. Biol. 19 (24), R1108-R1109.

Ehrsson, H., Spence, C., Passingham, R., 2004. That's my hand! Activity in premotor cortex reflects feeling of ownership of a limb. Science 305 (5685), 875-877.

Ehrsson, H., Holmes, N., Passingham, R., 2005. Touching a rubber hand: feeling of body ownership is associated with activity in multisensory brain areas. J. Neurosci. 25 (45), 10564-10573.

Ehrsson, H., Wiech, K., Weiskopf, N., Dolan, R., Passingham, R., 2007. Threatening a rubber hand that you feel is yours elicits a cortical anxiety response. Proc. Natl. Acad. Sci. 104 (23), 9828.

Ehrsson, H., Rosen, B., Stockselius, A., Ragno, C., Kohler, P., Lundborg, G., 2008. Upper limb amputees can be induced to experience a rubber hand as their own. Brain 131 (12), 3443-3452.

Fink, G., Marshall, J., Halligan, P., Frith, C., Driver, J., Frackowiak, R., 1999. The neural consequences of conflict between intention and the senses. Brain 122 (3), 497-512.

Fuchs, M., Kastner, J., Wagner, M., Hawes, S., Ebersole, J., 2002. A standardized boundary element method volume conductor model. Clin. Neurophysiol. 113 (5), 702-712.

Gallagher, S., 2000. Philosophical conceptions of the self: implications for cognitive science. J. Exp. Psychol. 24, 461-475.

Ganis, G., Keenan, J., Kosslyn, S., Pascual-Leone, A., 2000. Transcranial magnetic stimulation of primary motor cortex affects mental rotation. Cereb. Cortex 10 (2), $175-180$.

Gastaut, H., 1952. Electrocortographic study of the reactivity of rolandic rhythm. Rev. Neurol. 87, 176-182.

Gastaut, H., Bert, J., 1954. EEG changes during cinematographic presentation. Electroencephalogr. Clin. Neurophysiol. 6, 433.

Gerardin, E., Sirigu, A., Lehéricy, S., Poline, J., Gaymard, B., Marsault, C., 2000. Partially overlapping neural networks for real and imagined hand movements. Cereb. Cortex 10 (11), 1093-1104.

Gillihan, S., Farah, M., 2005. Is self special? A critical review of evidence from experimental psychology and cognitive neuroscience. Psychol. Bull. 131 (1), 76-97.

Goldman, R., Stern, J., Engel, J., Cohen, M., 2002. Simultaneous EEG and fMRI of the alpha rhythm. Neuroreport 13 (18), 2487-2492.

Grèzes, J., Decety, J., 2001. Functional anatomy of execution, mental simulation, observation, and verb generation of actions: a meta-analysis. Hum. Brain Mapp. 12 (1), 1-19.

Hägni, K., Eng, K., Hepp-Reymond, M.C., Holper, L., Keisker, B., Siekierka, E., Kiper, D.C., 2008. Observing virtual arms that you imagine are yours increases the galvanic skin response to an unexpected threat. PLoS One 3 (8), e3082.

Howe, R., Sterman, M., 1972. Cortical-subcortical EEG correlates of suppressed motor behavior during sleep and waking in the cat. Electroencephalogr. Clin Neurophysiol 32 (6), 681-695.

Ijsselsteijn, W., Kort, Y., Haans, A., 2006. Is this my hand I see before me? The rubber hand illusion in reality, virtual reality, and mixed reality. Presence Teleoperators Virtual Environ. 15, 455-464.

Ionta, S., Blanke, O., 2009. Differential influence of hands posture on mental rotation of hands and feet in left and right handers. Exp. Brain Res. 195 (2), 207-217.

Ionta, S., Ferretti, A., Merla, A., Tartaro, A., Romani, G., 2010. Step-by-step: the effects of physical practice on the neural correlates of locomotion imagery revealed by fMRI. Hum. Brain Mapp. 31 (5), 694-702.

Ionta, S., Perruchoud, D., Draganski, B., Blanke, O., 2012. Body context and posture affect mental imagery of hands. PLoS One 7 (3), e34382.

Ionta, S., Sforza, A., Funato, M., Blanke, O., 2012. Anatomically plausible illusory posture affects mental rotation of body parts. Cogn. Affective Behav. Neurosci. http:// dx.doi.org/10.3758/s13415-012-0120-z.

Jeannerod, M., 2006. What Actions Tell the Self. Oxford University Press, Oxford.

Jeannerod, M., 2007. Being oneself. J. Physiol. 101 (4-6), 161-168.

Kanayama, N., Sato, A., Ohira, H., 2007. Crossmodal effect with rubber hand illusion and gamma-band activity. Psychophysiology 44 (3), 392-402.

Kanayama, N., Sato, A., Ohira, H., 2009. The role of gamma band oscillations and synchrony on rubber hand illusion and crossmodal integration. Brain Cogn. 69 (1), 19-29.

Karnath, H.O., Baier, B., 2010. Right insula for our sense of limb ownership and selfawareness of actions. Brain Struct. Funct. 214, 411-417.

Kosslyn, S., Ganis, G., Thompson, W., 2001. Neural foundations of imagery. Nat. Rev. Neurosci. 2 (9), 635-642.

Lebedev, M., Nicolelis, M., 2006. Brain-machine interfaces: past, present and future. Trends Neurosci. 29 (9), 536-546.

Legrand, D., Ruby, P., 2009. What is self-specific? Theoretical investigation and critical review of neuroimaging results. Psychol. Rev. 116 (1), 252-282.

Lenggenhager, B., Tadi, T., Metzinger, T., Blanke, O., 2007. Video ergo sum: manipulating bodily self-consciousness. Science 317 (5841), 1096-1099.

Lenggenhager, B., Halje, P., Blanke, O., 2011. Alpha band oscillations correlate with illusory self-location induced by virtual reality. Eur. J. Neurosci. 1-9.

Lloyd, D., 2007. Spatial limits on referred touch to an alien limb may reflect boundaries of visuo-tactile peripersonal space surrounding the hand. Brain Cogn. 64 (1), 104-109.

Lloyd, D., Morrison, I., Roberts, N., 2006. Role for human posterior parietal cortex in visual processing of aversive objects in peripersonal space. J. Neurophysiol. 95 (1), $205-214$.
Makin, T., Holmes, N., Ehrsson, H., 2008. On the other hand: dummy hands and peripersonal space. Behav. Brain Res. 191 (1), 1-10.

Malouin, F., Richards, C., Jackson, P., Dumas, F., Doyon, J., 2003. Brain activations during motor imagery of locomotor-related tasks: a PET study. Hum. Brain Mapp. 19 (1), 47-62.

Marasco, P., Kim, K., Colgate, J., Peshkin, M., Kuiken, T., 2011. Robotic touch shifts perception of embodiment to a prosthesis in targeted reinnervation amputees. Brain $1-12$.

Maris, E., Oostenveld, R., 2007. Nonparametric statistical testing of EEG-and MEG-data. J. Neurosci. Methods 164 (1), 177-190.

Mercier, C., Aballea, A., Vargas, C.D., Paillard, J., Sirigu, A., 2008. Vision without proprioception modulates cortico-spinal excitability during hand motor imagery. Cereb. Cortex 18 (2), 272-277.

Michel, C.M., Murray, M.M., 2012. Towards the utilization of EEG as a brain imaging tool. Neuroimage 61 (2), 371-385.

Millán, J.D., Rupp, R., Müller-Putz, G.R., Murray-Smith, R., Giugliemma, C., Tangermann, M., Vidaurre, C., Cincotti, F., Kübler, A., Leeb, R., Neuper, C., Müller, K.R., Mattia, D., 2010. Combining brain-computer interfaces and assistive technologies: state-ofthe-art and challenges. Front. Neurosci. 161 (4), 1-15.

Miller, K.J., Shalk, G., Fetz, E.E., Den Nijs, M., Ojemann, J.G., Rao, R.P.N., 2010. Cortical activity during motor execution, motor imagery, and imagery-based online feedback. Proc. Natl. Acad. Sci. U.S.A. 107 (9), 4430-4435.

Mitsis, G.D., Lannetti, G.D., Smart, T.S., Tracey, I., Wise, R.G., 2008. Regions of interest analysis in pharmacological fMRI: how do the definition criteria influence the inferred result? Neuroimage 40 (1), 121-132.

Mukamel, R., Ekstrom, A., Kaplan, J., Iacoboni, M., Fried, I., 2010. Single-neuron responses in humans during execution and observation of actions. Curr. Biol. 20 (8), 750-756.

Mulert, C., Lorenz, J., Schmitt, R., Bussfeld, P., Pogarell, O., Möller, H.J., Juckel, G., Hegerl, U., 2003. Integration of fMRI and simultaneous EEG: towards a comprehensive understanding of localization and time-course of brain activity in target detection. Neuroimage 22 (1), 83-94.

Munzert, J., Lorey, B., Zentgraf, K., 2009. Cognitive motor processes: the role of motor imagery in the study of motor representations. Brain Res. Rev. 60 (2), 306-326.

Navarro, X., Krueger, T.B., Lago, N., Micera, S., Stieglitz, T., Dario, P., 2005. A critical review of interfaces with the peripheral nervous system for the control of neuroprostheses and hybrid bionic systems. J. Peripher. Nerv. Syst. 10 (3), 229-258.

Neuper, C., Müller-Putz, G., Scherer, R., Pfurtscheller, G., 2006. Motor imagery and EEGbased control of spelling devices and neuroprostheses. Prog. Brain Res. 159, 393-409.

Neuper, C., Reinhold, S., Wriessnegger, S., Pfurtscheller, G., 2009. Motor imagery and action observation: modulation of sensorimotor brain rhythms during mental control of a brain-computer interface. Clin. Neurophysiol. 120 (2), 239-247.

Nichols, T., Holmes, A., 2002. Nonparametric permutation tests for functional neuroimaging: a primer with examples. Hum. Brain Mapp. 15 (1), 1-25.

Niedermeyer, E., Lopes da Silva, F., 1993. Electroencephalography: Basic Principles, Clinical Applications and Related Fields, 3rd ed. Williams and Wilkins, Baltimore.

Northoff, G., Heinzel, A., de Greck, M., Bermpohl, F., Dobrowolny, H., Panksepp, J., 2006. Self-referential processing in our brain-a meta-analysis of imaging studies on the self. Neuroimage 31 (1), 440-457.

Oakes, T., Pizzagalli, D., Hendrick, A., Horras, K., Larson, C., Abercrombie, H., 2004. Functional coupling of simultaneous electrical and metabolic activity in the human brain. Hum. Brain Mapp. 21 (4), 257-270.

Oberman, L., Hubbard, E., McCleery, J., Altschuler, E., Ramachandran, V., Pineda, J., 2005. EEG evidence for mirror neuron dysfunction in autism spectrum disorders. Cogn. Brain Res. 24 (2), 190-198.

Ocklenburg, S., Ruther, N., Peterburs, J., Pinnow, M., Gunturkun, O., 2010. Laterality in the rubber hand illusion. Laterality $1-14$.

Overney, L., Blanke, O., 2009. Impaired imagery for upper limbs. Brain Topogr. 22 (1), 27-43.

Parsons, L., Fox, P., Downs, J., Glass, T., Hirsch, T., Martin, C., 1995. Use of implicit motor imagery for visual shape discrimination as revealed by PET. Nature 375 (6526), 54-58.

Pascual-Marqui, R., 2002. Standardized low-resolution brain electromagnetic tomography (sLORETA): technical details. Methods Find. Exp. Clin. Pharmacol. 24, 5-12.

Peled, A., Pressman, A., Geva, A.B., Modai, I., 2003. Somatosensory evoked potentials during a rubber-hand illusion in schizophrenia. Schizophr. Res. 64 (2), 157-163.

Pfurtscheller, G., 1981. Central beta rhythm during sensorimotor activities in man. Electroencephalogr. Clin. Neurophysiol. 51 (3), 253-264.

Pfurtscheller, G., Neuper, C., 1994. Event-related synchronization of mu rhythm in the EEG over the cortical hand area in man. Neurosci. Lett. 174 (1), 93-96.

Pfurtscheller, G., Neuper, C., 1997. Motor imagery activates primary sensorimotor area in humans. Neurosci. Lett. 239 (2-3), 65-68.

Pfurtscheller, G., Neuper, C., 2001. Motor imagery and direct brain-computer communication. Proc. IEEE 89 (7), 1123-1134.

Pfurtscheller, G., Neuper, C., Andrew, C., Edlinger, G., 1997a. Foot and hand area mu rhythms. Int. J. Psychophysiol. 26 (1-3), 121-135.

Pfurtscheller, G., Neuper, C., Flotzinger, D., 1997b. EEG-based discrimination between imagination of right and left hand movement. Electroencephalogr. Clin. Neurophysiol. 642-651.

Pineda, J., 2005. The functional significance of mu rhythms: translating "seeing" and "hearing" into "doing". Brain Res. Rev. 50 (1), 57-68.

Press, C., Heyes, C., Haggard, P., Eimer, M., 2008. Visuotactile learning and body representation: an ERP study with rubber hands and rubber objects. J. Cogn. Neurosci. 20 (2), 312-323. 
Rohde, M., Di Luca, M., Ernst, M., 2011. The rubber hand illusion: feeling of ownership and proprioceptive drift do not go hand in hand. PLoS One 6 (6).

Schaefer, M., Flor, H., Heinze, H.-J., Rotte, M., 2006a. Dynamic modulation of the primary somatosensory cortex during seeing and feeling a touched hand. Neuroimage 29 (2), 587-592.

Schaefer, M., Noennig, N., Heinze, H.-J., Rotte, M., 2006b. Fooling your feelings: artificially induced referred sensations are linked to a modulation of the primary somatosensory cortex. Neuroimage 29 (1), 67-73.

Schaefer, M., Flor, H., Heinze, H.J., Rotte, M., 2007. Morphing the body: illusory feeling of an elongated arm affects somatosensory homunculus. NeuroImage 36 (3), 700-705.

Schütz-Bosbach, S., Mancini, B., Aglioti, S.M., Haggard, P., 2006. Self and other in the human motor system. Curr. Biol. 16 (18), 1830-1834.

Schütz-Bosbach, S., Avenanti, A., Aglioti, S.M., Haggard, P., 2009. Don't do it! Cortical inhibition and self-attribution during action observation. J. Cogn. Neurosci. 21 (6), 1215-1227.

Sirigu, A., Duhamel, J., Cohen, L., Pillon, B., Dubois, B., Agid, Y., 1996. The mental representation of hand movements after parietal cortex damage. Science 273 (5281), 1564-1568.

Slater, M., Perez-Marcos, D., Ehrsson, H., Sanchez-Vives, M., 2008. Towards a digital body: the virtual arm illusion. Front. Hum. Neurosci. 2, 6.

Tadi, T., Overney, L.S., Blanke, O., 2009. Three sequential brain activations encode mental transformations of upright and inverted human bodies: a high resolution evoked potential study. Neuroscience 159, 1316-1325.
Tremblay, C., Robert, M., Pascual-Leone, A., Lepore, F., Nguyen, D., Carmant, L., 2004 Action observation and execution: intracranial recordings in a human subject. Neurology 63 (5), 937-938.

Tsakiris, M., 2010. My body in the brain: a neurocognitive model of body-ownership. Neuropsychologia 48 (3), 703-712.

Tsakiris, M., Haggard, P., 2005. The rubber hand illusion revisited: visuotactile integration and self-attribution. J. Exp. Psychol. Hum. Percept. Perform. 31 (1), 80-91.

Tsakiris, M., Hesse, M., Boy, C., Haggard, P., Fink, G., 2007. Neural signatures of body ownership: a sensory network for bodily self-consciousness. Cereb. Cortex 17 (10), 2235-2244.

Tsakiris, M., Carpenter, L., James, D., Fotopoulou, A., 2009. Hands only illusion: multisensory integration elicits sense of ownership for body parts but not for noncorporeal objects. Exp. Brain Res. 204 (3), 343-352.

Vallar, G., Ronchi, R., 2009. Somatoparaphrenia: a body delusion. A review of the neuropsychological literature. Exp. Brain Res. 192 (3), 533-551.

Vogeley, K., Fink, G., 2003. Neural correlates of the first-person-perspective. Trends Cogn. Sci. 7 (1), 38-42.

Wolbers, T., Weiller, C., Büchel, C., 2003. Contralateral coding of imagined body parts in the superior parietal lobe. Cereb. Cortex 13 (4), 392-399.

Wolpaw, J., McFarland, D., 2004. Control of a two-dimensional movement signal by a noninvasive brain-computer interface in humans. Proc. Natl. Acad. Sci. U.S.A. 101 (51), 17849

Zeller, D., Gross, C., Bartsch, A., Johansen-Berg, H., Classen, J., 2011. Ventral premotor cortex may be required for dynamic changes in the feeling of limb ownership: a lesion study. J. Neurosci. 31 (13), 4852-4857. 